\title{
Ultrasound-Mediated Drug/Gene Delivery in Solid Tumor Treatment
}

\author{
Yufeng Zhou* \\ Division of Engineering Mechanics, School of Mechanical and Aerospace \\ Engineering, Nanyang Technological University, Singapore
}

Submitted July 2012. Accepted for publication December 2012.

\begin{abstract}
Ultrasound is an emerging modality for drug delivery in chemotherapy. This paper reviews this novel technology by first introducing the designs and characteristics of three classes of drug/gene vehicles, microbubble (including nanoemulsion), liposomes, and micelles. In comparison to conventional free drug, the targeted drug-release and delivery through vessel wall and interstitial space to cancerous cells can be activated and enhanced under certain sonication conditions. In the acoustic field, there are several reactions of these drug vehicles, including hyperthermia, bubble cavitation, sonoporation, and sonodynamics, whose physical properties are illustrated for better understanding of this approach. In vitro and in vivo results are summarized, and future directions are discussed. Altogether, ultrasound-mediated drug/gene delivery under imaging guidance provides a promising option in cancer treatment with enhanced agent release and site specificity and reduced toxicity.
\end{abstract}

Keywords: ultrasound, drug/gene delivery, drug vehicle, bubble cavitation, sonoporation

\section{INTRODUCTION}

Cancer is currently the second leading cause of mortality in the world. According to the Surveillance Epidemiology and End Results (SEER) Cancer Statistics Review, there were more than 570,000 deaths due to cancer and approximately 1.6 million new cases diagnosed in 2011 in the United States [1]. Although half of cancer patients die as a result of malignant metastases, lack of control of the primary tumor causes a critical failure, especially for cancers at the cervix, colon, ovaries, pancreas, and brain. Extensive research and substantial progress in the development of anti-cancer agents have been made in recent decades. However, success achieved in solid tumors (accounting for $85 \%$ of all cancers) treatment is not satisfactory. Three physiological barriers limit the efficacy and safety of chemo- and bio-therapeutics especially for the most promising macromolecular agents (i.e., monoclonal antibodies, cytokines, antisense oligonucleotides, and genes): blood vessel walls (vasculature occupying $1-10 \%$ of the tumor volume); collagen-rich interstitial space accounting for a large

*Corresponding author: Yufeng Zhou, Nanyang Technological University, School of Mechanical and Aerospace Engineering, Division of Engineering Mechanics, 50 Nanyang Avenue, Singapore, 639798. Phone: (65) 6790-4482. Fax: (65) 6792-4062. E-mail: yfzhou@ntu.edu.sg. 
volume of tumors; and the cancer cell membrane, due to the heterogeneity of antigen and receptor expression for affinity-targeted delivery of drugs [2]. The typical diffusion time required for macromolecules to cross a distance of $200 \mu \mathrm{m}$ (average distance between tumor capillaries) is longer than their average half-life time [3]. Uneven and slowed blood flow within tumors because of abnormal (aberrant branching and tortuosity), heterogeneous, and inefficient distribution of a vasculature [3], high interstitial pressures due to the tumor vessels' leakage, the absence of a functional lymphatic system [4], and fibrillar collagen in the extracellular matrix [5] further complicate effective and uniform delivery of high-molecular-weight (molecular weight, MW > $2000 \mathrm{Da}$ ) drugs. The dose of chemo- and radio-therapeutic agents for clinical therapy usually destroys normal cells/tissues, and result in a variety of undesirable side effects, including cardiotoxicity, immune suppression, and nephrotoxicity $[6,7]$.

In order to overcome this stalemate, various approaches have been explored to maximize drug localization to the tumor while minimizing systemic toxicity [8]. Lately, controlling drug deposition and release in the region-of-interest by an external signal, such as light, neutron beam, magnetic field, or mechanical energy, is gaining attention [9]. Ultrasound has been applied in medical diagnosis since World War II. In recent years, it has taken on a new life in therapeutic applications [10, 11], such as targeting or controlling drug release $[12,13]$. In 1976, the cytotoxic effect of nitrogen mustard on mouse leukemia L1210 after sonication without any mechanical damage to cells was first observed, which cannot be explained merely by ultrasound-induced hyperthermia [10]. This approach is highly attractive because of wide acceptance of ultrasound in medicine, easy and accurate focusing to the deeply seated organs in the body, high efficiency in perturbing cell membranes and increasing their permeability, noninvasiveness, non-viral nature, low cost, and non-ionization for theoretically unlimited treatment [11]. Meanwhile, there is no effect on the non-sonicated region. Overall, this strategy provides an option in treating the localized tumors that may be inoperative by surgery either physiologically or cosmetically.

In this paper, the progress and characteristics of ultrasound-mediated drug delivery in the solid tumor treatment are reviewed. First, the design of an acoustically-active drug, such as microbubble, nanoemulsion, liposome, and micelle, is discussed. Then the underlying mechanisms of ultrasound-induced enhancement of drug delivery are explained. In vitro and in vivo outcomes of this novel technology are summarized for future translation into clinics. Finally, technical challenges and the potential direction of development are discussed.

\section{ACOUSTICALLY-ACTIVE DRUG VEHICLES}

The most popular vehicles activated by sonication include microbubbles, nanoemulsions, liposomes, and polymeric micelles, which have a promising enhancement of localized delivery in solid tumors up to 5 to 10 -fold over traditional methods of delivery [14].

\subsection{Microbubbles}

Ultrasound contrast agents (UCAs), biocompatible microbubbles less than $10 \mu \mathrm{m}$ in size in order to exit the heart through the pulmonary capillaries, are popular in perfusion monitoring to measure vascular density and microvascular flow rate [15]. A typical dose 
of UCAs for an echocardiographic evaluation is $10^{9}-10^{10}$ microbubbles in a $1-2 \mathrm{~mL}$ bolus intravenous injection [16]. Microbubbles have a gas core (i.e., perfluoropentane, sulfur hexafluoride, nitrogen) and a highly cohesive and insoluble shell (i.e., protein, phospholipid, polymer), permitting prolonged circulation, and preventing nonspecific removal from the circulation by the reticuloendothelial system (RES). The gas core not only has a significant acoustic impedance mismatch for strong echogenicity, but also is compressible for bubble cavitation (both stable and inertial cavitation, depending upon whether the acceleration terms are dominated by the pressure of the gas or the inertia of the inrushing liquid). For stable cavitation (SC), a bubble oscillates nonlinearly around its equilibrium radius. The stable pulsation of fluid produces microstreaming and shear stress, which are shown to induce transient compromise of cell membranes. However, in inertial cavitation (IC), a bubble is reduced to a minute fraction of its original size, and the gas within dissipates into the surrounding liquid via a violent mechanism. UCAs, regardless if being co-administered or encapsulated with pharmaceutical agents, can be intentionally ruptured by ultrasonic waves at a moderately high acoustic pressure at the target sites [17, 18].

\subsubsection{Drug-loaded Microbubbles}

There are various ways of entrapping drugs within a microbubble (Figure 1) [20, 21]. Drugs may be incorporated into the membrane or in a shell of microbubbles. Stable and

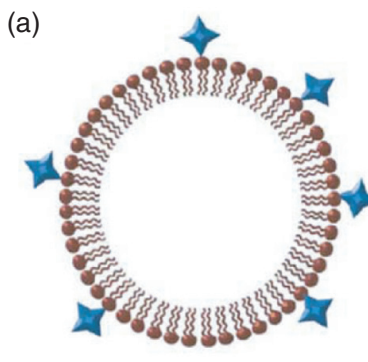

(d) (b)

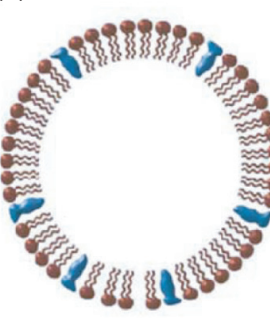

(c)

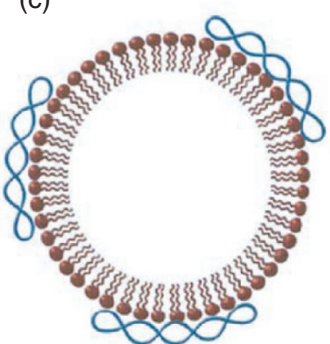

(e)
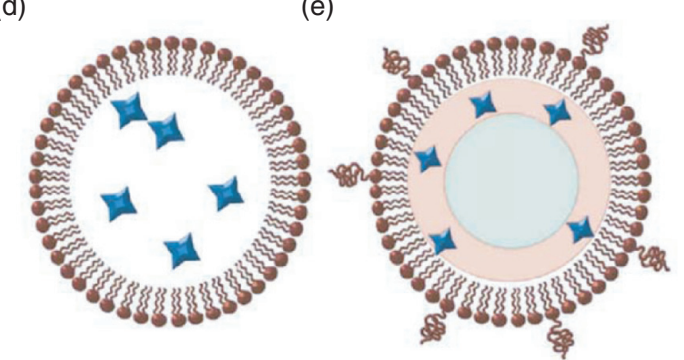

Figure 1. Different approaches of loading drug/DNA into microbubbles by (a) attaching to the membrane, (b) embedding within the membrane, (c) bounding non-covalently to the surface, (d) enclosing inside, and (e) incorporating into an oily film surrounded by a stabilizing layer with a ligand for targeting (used with permission [19]). 
strong deposition of the charged drugs in or onto the microbubbles' shell is realized by electrostatic interactions. However, Kupfer cells, leukocytes, and macrophages have the tendency to capture charged microbubbles, which could substantially decrease their half-life. In addition, drugs can also be embedded into microbubbles. The advantages of drug-loaded microbubbles are that the loaded-drugs can be released at the occurrence of IC into the tissue due to the induced shock wave, microstreaming and microjet. Meanwhile, the process could be tracked with sonography as the drug carriers are essentially UCAs. However, the monolayer lipid shell (a few $\mathrm{nm}$ ) limits loading the hydrophobic pharmaceuticals, and may have a premature release. Although a thicker triglyceride lipid shell and dissolved drug is a solution, it is only available for hydrophobic drugs (i.e., paclitaxel). Biocompatible and biodegradable polymeric (i.e., pLGA) microbubbles possess a thick and rigid shell for a prolonged circulation halflife, and permit a much higher loading capacity of both hydrophobic and hydrophilic drugs whose release rate depends on the drug properties (i.e., lipophilicity and water solubility).

The aforementioned methods are most applicable to highly active drugs, such as gene-based drugs, assuring protection of the drug and lowering the propensity of premature release. Therapeutic genes with several thousand pairs and MW over 1 million Da cannot cross the capillary fenestrations of blood vessels [20]. In addition, intravenous administration is not suitable for genetic materials because of the rapid metabolization by serum esterases. After reaching the tissue targets, genes must penetrate cell membranes, pass cytoplasm, enter the cells' nuclei, and then be digested by lysosomes within the cells. Drawbacks of incorporated naked plasmid DNA (pDNA) and pDNA-polymer complexes released from microbubbles are (a) large microbubble size (3-7 $\mu \mathrm{m}$ for consequent short circulation time and ineffective extravasation into the tumor); (b) the necessity to complex the pDNA with cationic polymers to prevent degradation during fabrication; (c) low loading efficiency of pDNA ( 6700 molecules/bubble) due to the limited number of cationic lipids; and (d) prematurely release of more than $20 \%$ of the encapsulated pDNA [22].

\subsubsection{Targeted Microbubbles}

Although acoustic radiation force could promote the attachment of microbubbles to endothelial cells in the wave propagation path, it is more attractive to selectively adhere microbubbles to cellular epitopes and receptors of cancer or solid tumors by one or several specific ligands, such as antibodies, carbohydrates, and/or peptides (Figure 2) [9]. Monoclonal antibodies have a very high specificity and selectivity to a large range of epitopes. In contrast, peptides are low-cost and less immunogenic because of much smaller size (5-15 amino acids). Significant enhancements of microbubbles adhesion to activated endothelium [24], rejecting tissues [25], neovasculature endothelium [26], lymph node-related vasculature [27], or activated platelets [28] have been reported. Simultaneous targeting to multiple ligands could synergistically increase adhesion strength [29]. There are two ways of coupling ligands to the microbubble shell: covalent (being attached to the head of phospholipids directly or via an extended polymer spacer arm), and non-covalent by avidin-biotin bridging because of the wide availability and excellent affinity. However, since avidin carries a strong positive charge in the 
(a)

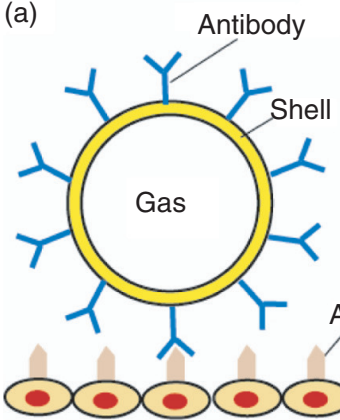

(b)

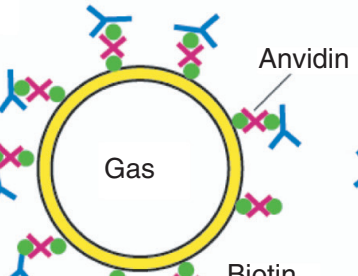

(c)<smiles>CC(C)[SeH][SeH]=[GeH2]</smiles>

Figure 2. Targeting microbubble to cancer cells by connecting the receptors on the surface with (a) an antibody, (b) an avidin bridge, and (c) a flexible spacer arm (used with permission [23]).

glycosylate layer, the bio-distribution of microbubbles may be altered, resulting in nonspecific adhesion and introduction of undesired immune response. In comparison, Streptavidin may be a better bridging molecule. It is found that connecting an intermediary spacer arm with the ligand at the end as a polyethylene glycol (PEG) molecular tether to the lipid shell indirectly is feasible, and has high specificity and targeting. Meanwhile, the tethered ligand could also be buried in a polymeric "overbrush" [30]. After sonication, the ligand is instantly revealed for targeting to avoid the undesired non-specific binding and uptake by RES.

Altogether, microbubbles can enhance transport of drugs/genes across vessel walls and cell membranes by the localized bubble cavitation. However, their disadvantages are the relatively low payload, and short circulation half-life (minutes for lipid-shelled microbubbles, although longer times for the polymer types).

\subsection{Nanoemulsions}

Although the use of microbubbles is very attractive in drug delivery, especially for its uniqueness of combined diagnosis and targeted therapy with a high effectiveness-tocost ratio, it lacks an essential prerequisite for effective extravasation into tumor and subsequent drug targeting with a sufficient lifetime in the circulation, because the pore size of most tumors is usually smaller $(380-780 \mathrm{~nm})$ [32]. An alternative solution to aforementioned problems is developing drug-loaded nanoparticles that could accumulate in tumor and then expand to microbubbles in situ under sonication, such as copolymer-stablilized echogenic perfluoropentane (PFP) nanoemulsions. The nanoemulsions are produced from drug-loaded poly(ethylene oxide)-co-poly(L-lactide) (PEG-PLLA) or poly(ethylene oxide-co-polycaprolactone (PEG-PCL) micelles. Dodecafluoropentane (DDFP) droplet remains as a liquid at the body temperature although it has a boiling temperature of $29^{\circ} \mathrm{C}$ at atmospheric pressure, but vaporizes under ultrasound exposure. Acoustic droplet vaporization (ADV), transiting droplet to bubble, is determined by a certain pressure threshold [33, 34]. The ADV threshold for PEG-PCL stabilized droplets is lower than that required by PEG-PLLA droplets. The 


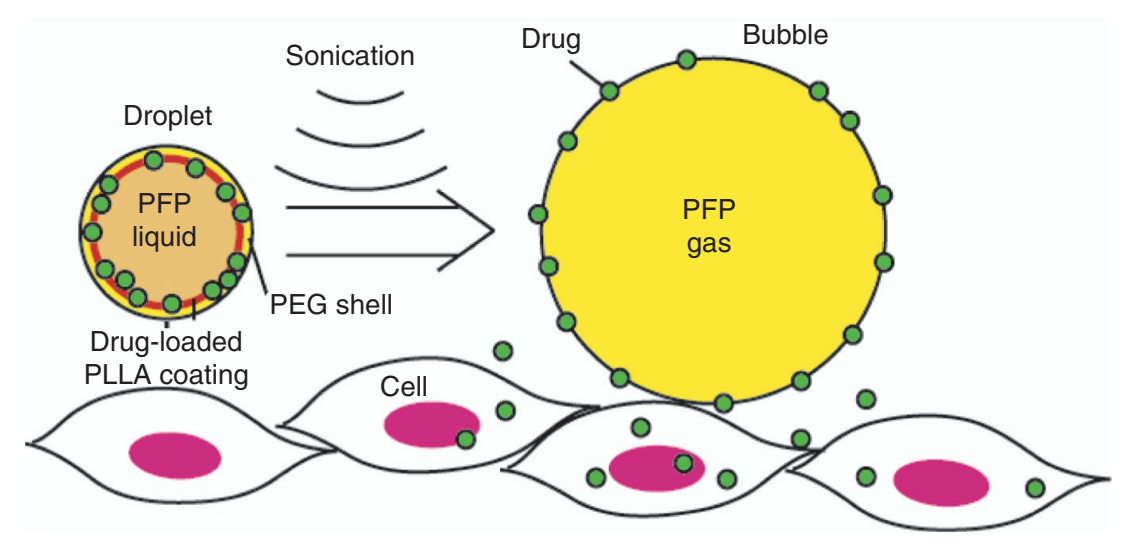

Figure 3. Schematic diagram of phase transition from nanodroplets to microbubbles, and consequent drug release into the neighboring cells under sonication (used with permission [31]).

higher echogenicity of microbubbles enables its discrimination from droplet's status in sonography. To prevent excessive phase transition in vasculature, infusion or injection through a low-gauge needle should be carried out in the application of PFP nanoemulsions. A significant volume expansion in the complete vaporization of the droplet and consequent shrinkage of the microbubble shell may rip off a drug to the local cells (Figure 3). All aspects considered, this method allows the enhancement of intracellular drug delivery with high ultrasound contrast and drug loading [31, 33, 34].

\subsection{Liposomes}

Liposomes with a typical diameter of 65-120 nm are non-toxic, biodegradable, and nonimmunogenic drug delivery vehicles for both hydrophilic and lipophilic drugs, such as doxorubicin (DOX) and vincristine [35, 36]. Local heating causes the liposome to change from a well-ordered gel to a less-structured liquid crystalline state by inducing thermotropic phase transitions on phospholipids (Figure 4). As a result, the cargo could be released with significant reduction on the systemic toxicity, and premature degradation or inactivation [37]. The in vivo stability and accumulation at the tumor site can be enhanced to 50 to 100-fold compared to the free drug [37], and small polymeric carriers can respond to both mechanical (i.e., fracture) and thermal (i.e., temperature elevation) activation. PEG liposomes containing DOX have been used in the treatment of Kaposi's sarcoma, refractory ovarian cancer, and breast cancer [38]. Furthermore, liposomes are also efficient as non-viral gene carriers [22].

Different strategies are applied for both drug loading and release from microbubbles and liposomes. Although the gas core occupies the greater volume of a microbubble, the drug loading capability can be enhanced by shell construction layer-by-layer or 

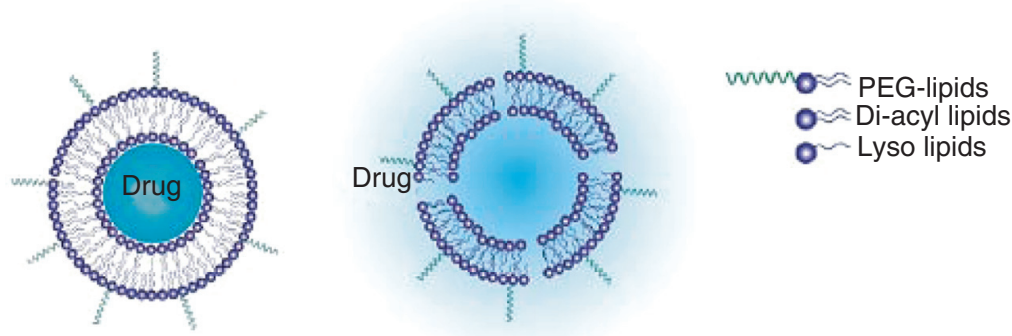

Figure 4. Temperature-sensitive liposomes (left) below and (right) above their phase transition temperature, which is typically chosen near $40^{\circ} \mathrm{C}$ for temperature-sensitive vehicles. Typical liposome diameter is $65-120 \mathrm{~nm}$ (used with permission [41]).

conjugation of drug-entrapped particles. In comparison, drug-specific loading techniques are applied to construct the core of a liposome. Drug release from microbubbles is mainly due to the ultrasound-induced IC, whereas that from a liposome is the consequence of the heat produced by the absorption of acoustic energy. Meanwhile, the gas nuclei will expand and dilate the monolayer boundary or the adjacent bilayer in the strong acoustic field. If the bubble expansion-induced stress is beyond the elastic threshold of the bounding membrane, the liposome will rupture, and then the incorporated contents will be released.

\subsubsection{Thermosensitive Liposomes}

The thermally responsive macromolecular carrier, poly( $\mathrm{N}$-isopropylacrylamide, NIPAAm), can be cheaply and conveniently synthesized by the approach of free radical polymerization [39]. However, precisely controlled release of chemo- and radiotherapeutic agents conjugated to its structure is difficult. Artificial Elastin-like polypeptides (ELPs) are genetically encodable, and polypeptide-based polymers that are recombinantly synthesized in E. Coli by over-expression of a synthetic gene, allowing control over the sequence, chain length, and the number and location of reactive side chains (e.g., lysine, cysteine) on the polypeptide [40]. ELPs exhibit lower critical solution temperature (LCST) than poly(NIPAAm). Traditional thermo-sensitive liposomes (TSLs) are triggered at $42-45^{\circ} \mathrm{C}$ for drug release over $\sim 30 \mathrm{~min}$. In comparison, the payload from low temperature-sensitive liposomes (LTSL) could be released in the temperature ranges of $39-40^{\circ} \mathrm{C}$, as shown in Figure 4.

\subsubsection{Non-thermal Liposomes}

Acoustically active lipospheres (AALS) do not rupture as easily as UCAs because the thicker shell retards the wall velocity in the bubble cavitation. A targeting ligand is necessarily attached to the liposomes to prevent them from being flushed away by the circulating blood. However, a lower paclitaxel loading efficiency $(4 \mathrm{mg} / \mathrm{mL}$ of 
microbubbles) may be an unfavorable side effect of the ligand attachment. Loading and release of hydrophobic drugs in AALS could be improved by varying the amount of oil and perfluorocarbon gas. In summary, it remains rather difficult to obtain an efficient drug release from AALS and to also incorporate hydrophilic drugs [42]. Meanwhile, air-filled echogenic liposomes (ELIPs) with an average size of $\sim 800 \mathrm{~nm}$ were developed to incorporate hydrophilic compounds inside lipsomes (Figure 5). Drug release from ELIPs is expected to occur relatively easily. When the expansion of ELIPs becomes so high that the elastic limit of the lipid shell is exceeded, drugs can leak out. The incorporation of short-chain lipids in the ELIPs shell results in a prolonged shell opening and a better drug release [42]. Because of the flexibility of the lipid monolayer, the ELIPs shell can reseal. Therefore, successive expansion cycles can be used to

(a)

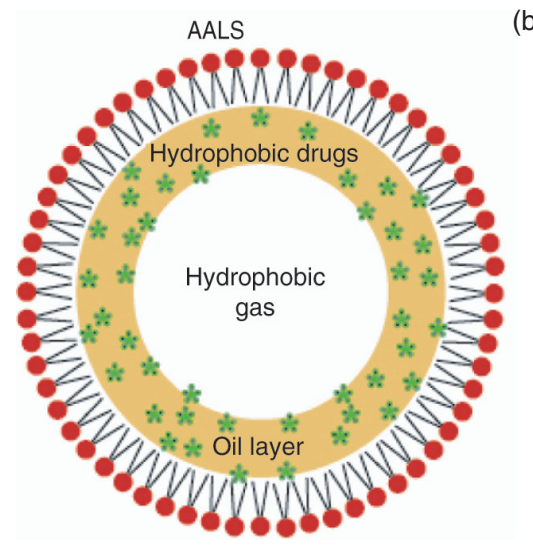

(b)

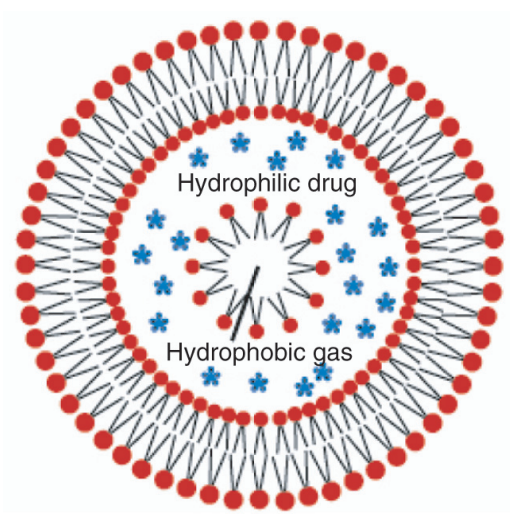

(c)

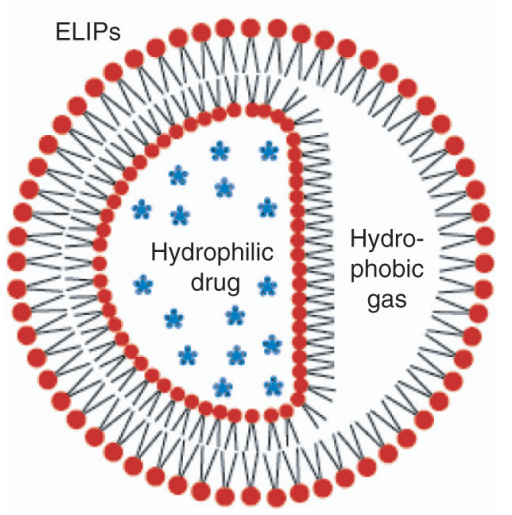

Figure 5. Schematics of (a) an acoustically active liposphere (AALS) encapsulating hydrophobic drugs and hydrophobic gas; and (b), (c) echogenic liposomes (ELIPs) encapsulating hydrophilic drugs and hydrophobic gas (used with permission [22]). 
improve drug release from ELIPs. AALS is conceptually similar to microbubbles, except the smaller size for easy extravasation, relatively low encapsulation efficiency, and limited drug release from the shell collapse because a thicker shell retards the wall velocity associated with IC.

\subsubsection{Liposomes Loaded Microbubble}

A more advanced drug-carrying microbubble was achieved by attaching both hydrophilic and hydrophobic drug-loaded nanoparticles to the structure via avidin-biotin interactions (Figure 6) [43-45]. As $\sim 10^{5}$ liposomes are bound to each microbubble, the drug loading capacity of microbubbles can be significantly enhanced. Attachment of polystyrene nanoparticles/liposomes onto the microbubble shell did not hinder IC. Cells close to liposome-loaded microbubbles contained liposome fragments after sonication. This concept can also be used to load siRNA and gene-containing

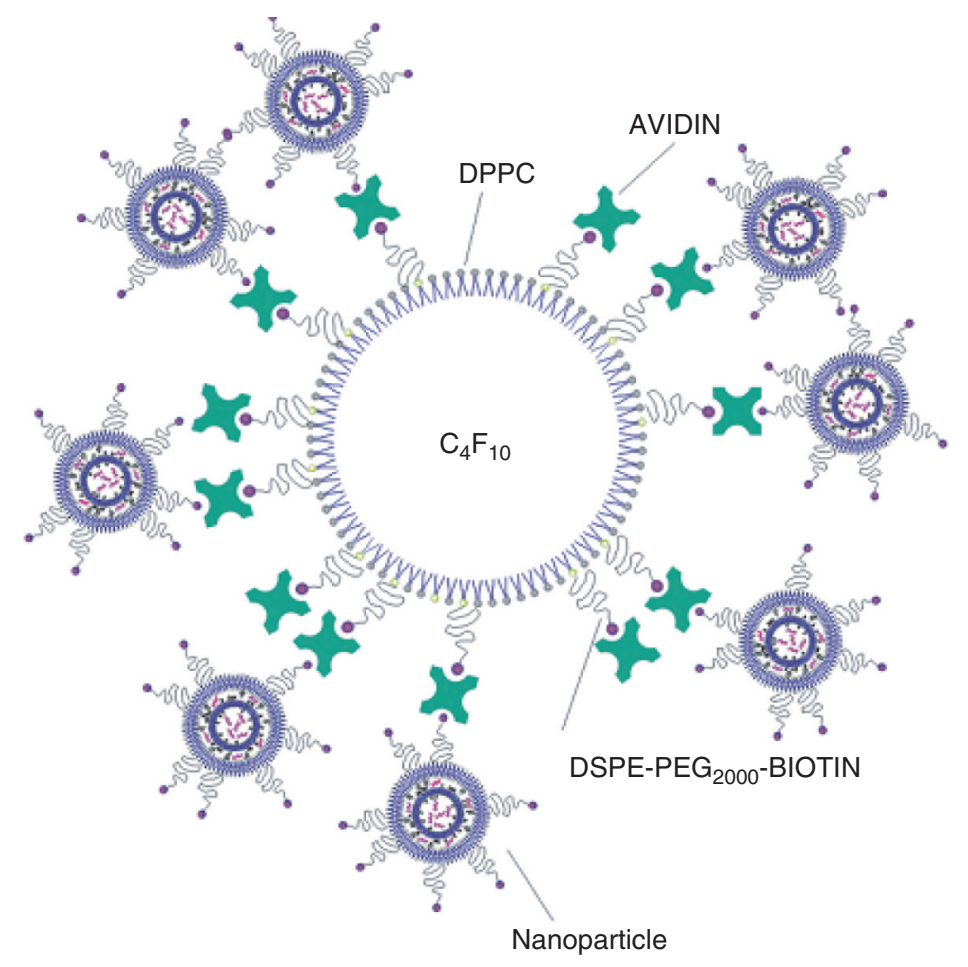

Figure 6. Schematic representation of a nanoparticles-loaded microbubble. PEGylated nanoparticles are attached to the microbubble with an avidinbiotin binding. Ultrasound irradiation results in the release of intact nanoparticles (used with permission [46]). DPPC: 1,2-Dipalmitoylsn-glycero-3-phosphorylcholine. DSPE: 1,2-Distearoyl-sn-glycero-3phosphorylethanolamine. 
liposomes (lipoplexes) to improve their transfection efficiency. These vehicles combine the advantages of liposomes and microbubbles and overcome individual limitations.

\subsection{Micelles}

A micelle is an aggregate of surfactant molecules dispersed in a liquid colloid with the hydrophilic "head" regions in contact with surrounding aqueous solution and the hydrophobic single-tail regions in the micelle center. They are small enough to avoid renal excretion, but permit extravasation at the tumor site via the enhanced penetration and retention (EPR) effect [47]. The structure of hydrophobic cores as drug reservoirs and hydrophilic shells ensure micelle solubility in the aqueous medium but prevent micelle aggregation. The PEG blocks with a typical length of 1-15 kDa prevent micelle opsonization, in order to avoid micelle recognition by RES. The thermodynamic and kinetic stability of micelle is determined by molecular interactions and the length of hydrophobic blocks and the micelle core type, respectively. Soluble polymeric carriers that undergo an LCST phase transition would enable targeting to heated tumors, and hydrophobically collapse and aggregate at temperatures higher than their LCST [40]. The self-assembly of amphiphilic block copolymers is activated thermodynamically and is reversible when the concentration reaches the critical micelle concentration (CMC). However, copolymer molecules are individual (unimers) at low concentrations. The encapsulated drug will be released from the prematurely destroyed micelles. The $\mathrm{CMC}$ is primarily controlled by the length of a hydrophobic block (the greater the length, the lower the $\mathrm{CMC}$ ), but less sensitive to the length of a hydrophilic block. However, an excessive concentration of the copolymer will initiate micelle aggregation and precipitation [48]. Micelles, whose glass transition temperature is higher than the physiological temperature (i.e., $37^{\circ} \mathrm{C}$ ), may survive for many hours or even days at concentration below the CMC. In contrast, it takes only a few minutes to dissociate micelles with a "soft" core (i.e., Pluronics). The CMC of this type of micelles is usually low because of direct mixing of the hydrophobic drugs with the soft core. The most outstanding attractions of micelles are the self-assembly of amphiphilic block copolymer molecules and drug encapsulation by simply mixing rather than conjugation. Bubble cavitation can occur, but only inside the micelle cores with an increased threshold [49]. In contrast, drug loading into micelles with solid cores requires a number of more complex techniques $[50,51]$.

\section{DRUG/GENE DELIVERY APPROACH}

The mechanisms for the ultrasound-mediated drug/gene delivery include both thermal and mechanical effects [52,53]. First, acoustic radiation force can help agents penetrate through the vessel wall to tissue. Second, ultrasound may affect the morphology and properties of the cell membrane due to bubble cavitation for drug permeation and absorption (sonoporation). Third, ultrasound-induced hyperthermia has a significant biological effect on cell activities and drug uptake. Last, ultrasound can alter the performance of a drug, for example, activating light-sensitive materials of hematoporphyrins to kill cancer cells and inhibit restenosis [54]. The efficiency of drug 
delivery is mainly determined by the acoustic intensity. However, with a higher power, the propensity of cell lysis is increased. Furthermore, cell membrane permeability and drug cytotoxicities can be enhanced at elevated temperatures [55].

\subsection{Acoustic Radiation Force}

Acoustic radiation force is associated with the propagation of acoustic waves through a dissipative medium, originating from a transfer of momentum from the wave (either from absorption or reflection) to the medium. Radiation force is proportional to the acoustic absorption and the rate of energy being applied, and is inversely proportional to the speed of sound in the medium. It could push drug-carrying nanoparticles toward a vessel's wall prior to fragmentation, and facilitate adhesion of them with receptorligands. Furthermore, the non-uniform displacement of tissue in the focal zone and transverse waves generated at the interface of tissue and fluid create a steep gradient in shear forces, where the resulting strain works on the cell-to-cell junctions and cellular interfaces. The penetration of various masses (small molecules, DNA, and nanoparticles) from the fluid into the adjacent epithelium could be increased both systemically and locally under sonication, as well as their rate of effective diffusion through the tissues. The increase in the intercellular gaps for enhanced local interstitial transport and reduction in interstitial fluid pressure in the core of tumors lead to improved extravasation of large molecules and consequent anti-tumor effects, which are correlated with more wide-spread induction of necrosis and apoptosis [17, 56, 57].

\subsection{Hyperthermia}

Hyperthermia induced by the absorption and dissipation of acoustic energy by tissue at the temperature of $40-45^{\circ} \mathrm{C}$ has a remarkable biological effect at the subcellular and cellular levels: decreased DNA synthesis, altered protein synthesis (i.e., heat shock proteins), disruption of the microtubule organizing center, varied expression of receptors and binding of growth factors, and changes in cell morphology and attachment $[58,59]$. Importantly, hyperthermia can also increase tumor blood flow and vascular permeability, which leads to its implementation for solid tumor therapy in combination with thermo-sensitive drugs. Even non-thermo sensitive polymeric carriers and drugs exhibit increased localization in heated tumors because of these physiological effects. Furthermore, when combined with chemo- and radio-therapy, hyperthermia produces more tumor cytotoxicity.

\subsection{Bubble Cavitation}

At the very low acoustic pressure [mechanical index $(\mathrm{MI})<\sim 0.1$ ], the microbubble oscillates symmetrically and linearly. At MI of $0.1-0.3$, the microbubble has more expansion than compression (nonlinear oscillations) while the shell still maintains its integrity, a state known as stable cavitation. Higher acoustic pressure of MI (0.3-0.6) forces microbubble oscillation for eventual destruction by either gas diffusion through compression or large shell defects through IC (Figure 7) [9, 61, 62]. However, the specific thresholds of different types of bubble oscillations vary a lot, depending on the bubble structure (i.e., initial size, wall thickness, and composition) and environmental 
(b)

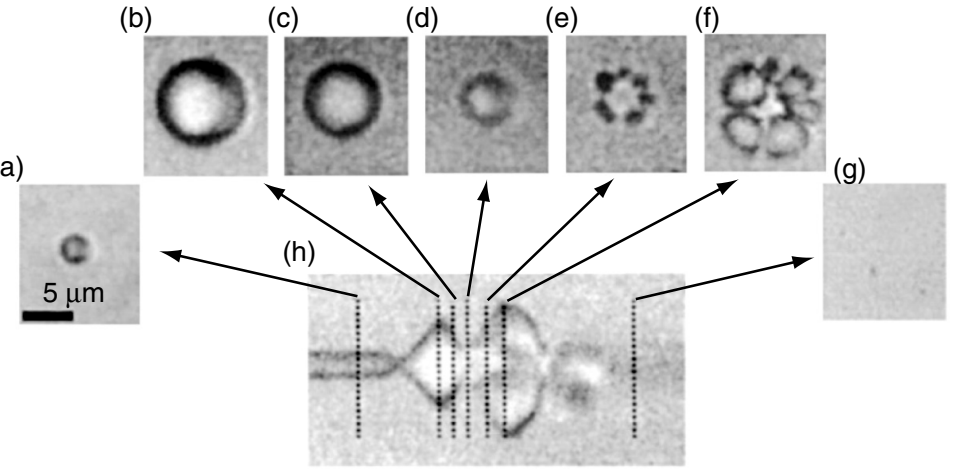

Figure 7. Optical frame images (a-g) and streak image (h, diameter of the bubble as a function of time) illustrating the oscillation and fragmentation of a microbubble. The bubble has an initial diameter of $3 \mathrm{~mm}$ (used with permission [60]).

conditions (i.e., ambient pressure and temperature). The microbubble wall reaches a velocity of several 100 to $1,000 \mathrm{~m} / \mathrm{s}$ at bubble collapse. Nano- or micro-particles, such as polymer particles or UCAs, can lower the cavitation threshold although inertial cavitation can also occur in most normal tissues. However, polymeric microbubbles illustrate different cavitation characteristics. At the low acoustic pressure, the polymeric microbubble will not oscillate actively because of the stiff shell. If acoustic pressure is beyond a critical value, defects or cracks will form in the shell for gas diffusion and microbubble fragmentation, although complete destruction is rare [63, 64].

Strong physical, chemical, and biological effects induced by both types of cavitation (SC and IC) are the mechanisms of microbubble-mediated drug delivery (Figure 8) [65-67]. Although shock waves, microstreaming, and microjet produced in the IC are short in time, the localized large pressure (i.e., $1000 \mathrm{~atm}$ ) can generate transient and nonlethal micropores in the endothelial cell, plasma, and nuclear membrane, making them more permeable by drugs [68]. During the bubble collapse, the local temperature can be as high as $5000 \mathrm{~K}$ (close to the surface temperature of the sun) and induce chemical changes in the medium (sonochemistry) [69]. The most significant is the generation of highly reactive oxygen species (ROS), such as free radicals, that can induce chemical transformations to permeabilize the cell membrane without producing pores on the cell membrane and affecting the cell viability. Meanwhile, endocytosis and phagocytosis in the uptake and fusions of lipid-shelled microbubbles are important in the intracellular drug absorption [9].

Blood has a higher viscosity than water, and consequently, a higher cavitation threshold (i.e., about 1.5 to 2 times of that in water at the frequency of $2.5 \mathrm{MHz}$ ). If nanoparticles (i.e., Polystyrene) are present in tumor, the cavitation threshold could be significantly lowered. Smaller particles have more effects on such a reduction in the cavitation threshold at the same concentration [22, 70]. However, polystyrene beads have a virtually indefinite lifetime in the body and are not FDA approved. 


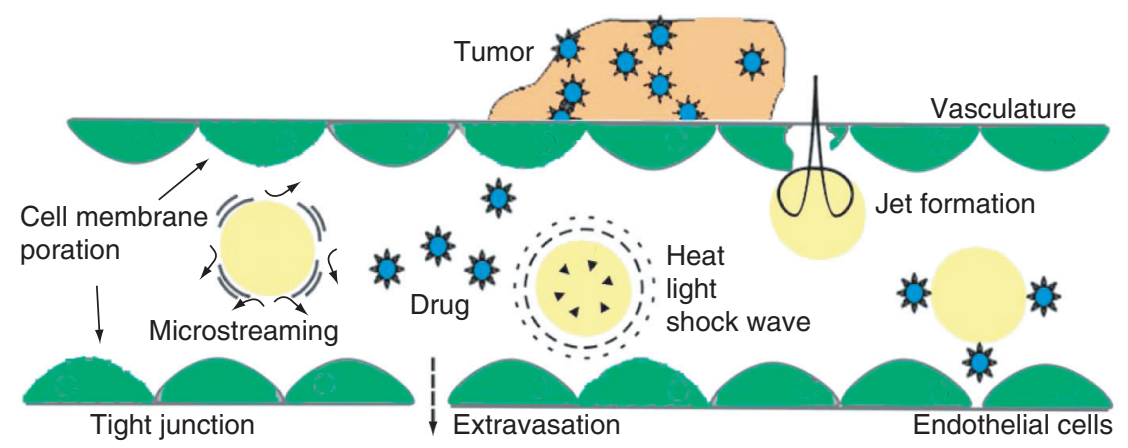

Figure 8. Schematic diagram of biological effects and drug release induced during bubble cavitation through the defective tumor microvasculature. Ultrasound can trigger drug release/activation locally by heat, light, shock wave, microstreaming, and microjet.

\subsection{Sonoporation}

Efficient and well-tolerated internalization of DNA and macromolecules with a diameter no more than $37 \mathrm{~nm}$ is allowed by the induction of transient and reversible holes in the cell membrane (sonoporation). The cell structure affects the sonoporation effect and the cell survivability, although the maximum size of molecules for entry as well as the opening period of the pores is not well known. Immediately after sonication, the MAT B III cells showed morphological changes (i.e., smoother surface and smaller size) because of the removal of macromolecules (i.e., glycoproteins or cell-surface receptor CD19) on the surface [71]. For the cells fixed during sonication, some large pores (on the order of $100 \mathrm{~nm}$ ) but not small ones (1-10 nm) can be produced, which may be due to the abundant microvilli on the cell surface, and the short duration of pore opening (Figure 9a). In comparison, fresh red blood cells have clear pores on their smooth surface after sonication (Figure 9b). Therefore, the pore size in sonoporation depends on both the ultrasound parameters and cell types. Small pores are more abundant on the cell membrane, facilitating the internalization of molecules whose amount is inversely proportional to their size, but reseals more quickly after the sonication. Large pores on the cell surface may not reseal, and $\sim 15 \mathrm{~s}$ disruption on a plasma membrane in smaller cells (i.e., fibroblasts and endothelial cells) is generally lethal. However, the nuclear membrane seems not affected by sonoporation, but nuclear complexes only allow the passive diffusion of small particles up to $9 \mathrm{~nm}$ [72-74]. Unfortunately, the leakage of cytosolic fluid could cause partial escape of internalized molecules. IC dominates in the ultrasound-mediated DNA transfection through sonoporation so that measuring the IC dose may be helpful in monitoring the delivery effect $[75,76]$.

\subsection{Sonodynamic Therapy}

Sonodynamic therapy (SDT), which uses a novel sonosensitive agent derived from chlorophyll that was originally used as light-activated chemicals for cancer therapy, is a promising cancer treatment modality [77, 78]. These sonosensitive drugs 
(a)

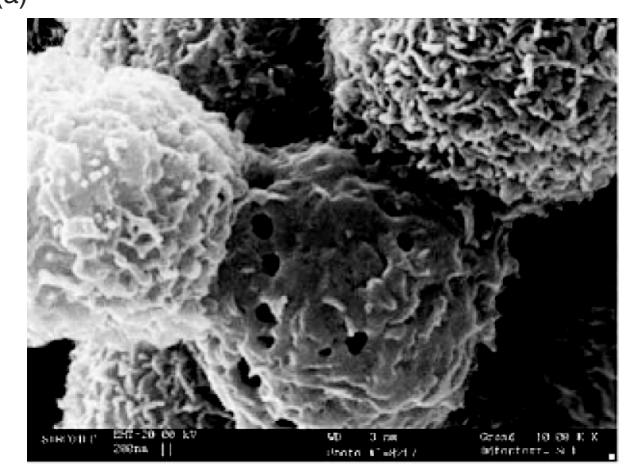

(b)

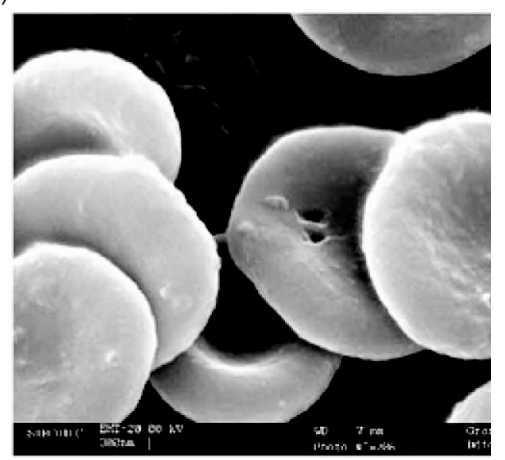

Figure 9. Representative pores at (a) MAT B III and (b) red blood cells after sonication $\left(f=2.25 \mathrm{MHz}, p^{-}=570 \mathrm{kPa}\right)$ in the presence of UCA (25 particles/cell for MAT B III and 1.2 particles/cell for red blood cells) by scanning electron microscope at a magnification of 10,000 (used with permission [71]).

(i.e., protoporphyrin IX), upon non-thermal sonication, create a short-lived cytotoxic ROS (i.e., superoxide radicals and singlet oxygen) to induce peroxidation of membrane lipids and irreversible alteration of the target tissue. It is assumed that sonication may introduce electronic excitation of porphyrins and a photochemical process in the cavitation process. The agents themselves have no antitumor ability but exhibit it only by the sonochemistry. Therefore, much less risk of adverse effects is expected on normal tissues.

In summary, ultrasound directly alters the biological or physiological properties of tissues facilitating transport and the bioavailability or efficacy of the drug or vehicle. It also produces changes in the surrounding tissue by indirect effects acting on the vehicle.

\section{IN VITRO AND IN VIVO RESULTS}

\subsection{Microbubble}

Early drug delivery studies involved the co-administration of a drug and microbubbles followed by sonication. Drug-loaded vehicles were then investigated for local release and cell uptake simultaneously only in the sonication area. Studies demonstrated that 20 $\mathrm{kHz}$ ultrasound irradiation in combination with the nanoparticle and chemotherapeutic agent 5-fluorouracil (FU) injections significantly suppressed tumor growth, and even complete elimination 2 months later, while the control volume increased and the tumor regrew from the peripheral points of the irradiated tumors $(p<0.05)$. Histological studies of H\&E stained sections of control tumor revealed viable tumor cells with well-defined nuclei. Treatment with 5-FU alone produced small necrotic regions in the tumor, most commonly near blood vessels. Ultrasound irradiation, in combination with 5-FU and polystyrene nanoparticles injection (100-280 $\mathrm{nm}$ in diameter and concentration up to $0.2 \% \mathrm{w} / \mathrm{w})$, resulted in dramatic tissue necrosis that was noted throughout the tumor [79]. 
Optimization of ultrasound frequency, intensity, duty cycle, time of irradiation, and concentration of Optison ${ }^{\circledR}$ led to $73.5 \pm 3.3 \%, 72.7 \pm 0.9 \%$, and $62.7 \pm 2.1 \%$ delivery of $10 \mathrm{kDa}, 70 \mathrm{kDa}$, and $2000 \mathrm{kDa}$ macromolecules in the MCF7 cell line, respectively, and $36.7 \pm 4.9 \%$ of cell transfection, while dead cell count was only $13.5 \pm 1.6 \%$ [80]. These results suggest that optimized treatment parameters provide efficient drug and gene delivery to cancer cells and could be utilized in further in vivo experiments (Figure 10).

Bubble cavitation-induced convection can transport drugs or particles over several $10 \mu \mathrm{m}$ from the vessel wall. The change in capillary permeation is strongly dependent on cavitation dose. Acoustic pressure of $0.75 \mathrm{MPa}$ at $1 \mathrm{MHz}$ can produce capillary rupture in the intact rat muscle microcirculation with high microbubble concentration, and the pressure required increases proportionally to the driving frequency. Combining

(a)

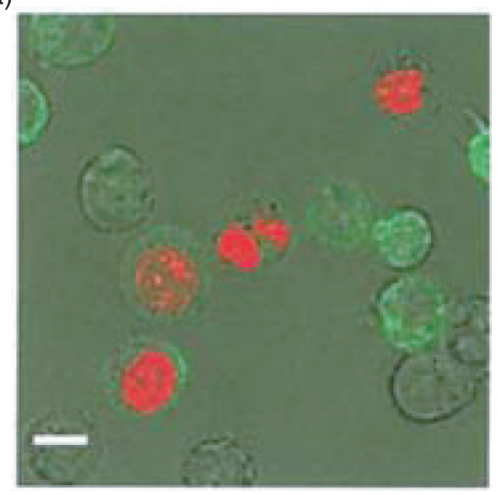

(c)

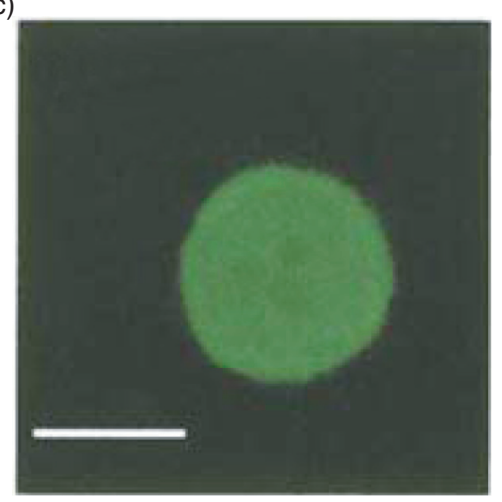

(b)

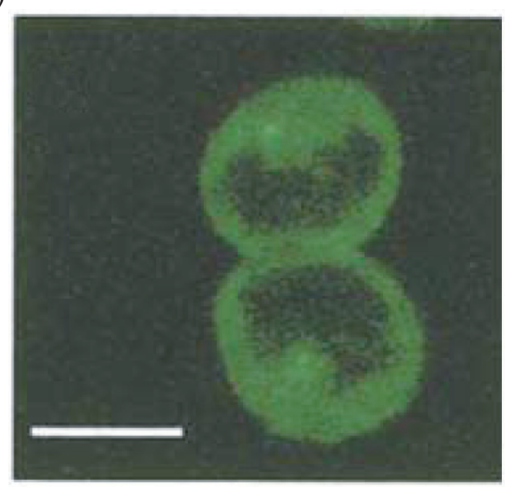

(d)

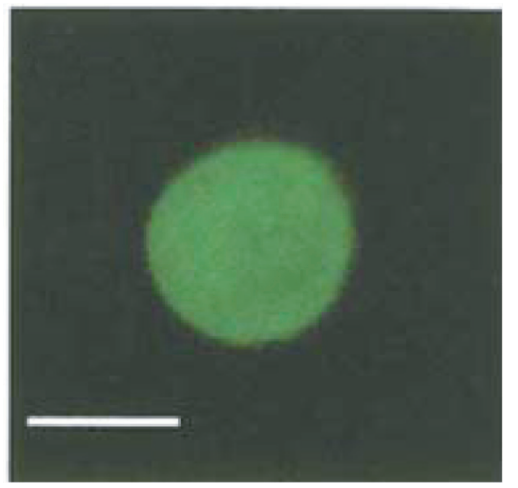

Figure 10. Confocal microscopy of MCF7 cells loaded with FITC-dextran by sonication ( $3 \mathrm{MHz}, 20 \%$ duty cycle, $3 \mathrm{~W} / \mathrm{cm}^{2}, 1$ minute with $200 \mu \mathrm{L}$ of Optison per $1 \mathrm{~mL}$ sample). (a) Red staining (propidium iodide) marks nuclei of dead or dying cells, while green indicates distribution of $70 \mathrm{kDa}$ FITC-dextran. Single cells loaded with (b) $2000 \mathrm{kDa}$, (c) $70 \mathrm{kDa}$, and (d) $10 \mathrm{kDa}$ FITC dextran. Bars indicate $10 \mathrm{~mm}$ (used with permission [80]). 
radiation force with destructive ultrasound pulses has increased deposition of oil on cell membranes in vitro by 10 folds in comparison to ultrasound alone [65].

Hemolysis, microvascular leakages, capillary ruptures, petechial hemorrhages, mild elevations of troponin-T in blood, cardiomyocyte injury, inflammatory cell infiltrations, and premature ventricular contractions were also observed in both in vitro and in vivo experiments after the sonication with UCAs [55]. The extent of these bioeffects is influenced by several factors, such as the concentration of UCAs, the drug-delivery method (intra-arterial vs. intravenous), the characteristics of acoustic field (i.e., frequency, pressure, beam size, duty cycle, exposure time), properties of the targeted tissue, and the ultrasound imaging mode (intermittent).

\subsection{Nanoemulsion}

Gadolinium-tagged nanoemulsion was found to accumulate in rabbit VX2 tumors for at least six hours after intravenous injection in $\mathrm{T}_{1}$-weighted MRI and change to microbubbles using short HIFU pulses in B-mode ultrasound image. As a result, HIFUinduced heating in the tumors are enhanced [81]. An ovarian carcinoma tumor disappeared completely after four sonications with systemic injections of the nanodroplet-encapsulated PTX, nbGEN (20 mg/kg as PTX), in two weeks. PTX alone is tightly retained by the nanodroplet carrier in vivo, which avoids the unintentional release to normal tissues. Thus, the unsonicated tumor grew at the same rate as control. A similar phenomenon was also found in the pancreatic cancer model [31]. It is important to note that the number of metastatic sites was substantially lower in the treated groups, indicating the potential ultrasound-induced immune response, whose mechanism is unclear and under investigation [31].

\subsection{Liposome}

Liposomes have a long half-life time in the circulation, resulting in a high-resolution positron emission tomography (PET) image of the vascular structure (Figure 11). The accumulation of liposomes with a vascular targeting ligand on the shell at the heart endothelium can occur quickly (i.e., within $100 \mathrm{~s}$ in mouse heart). In comparison, lipidshelled microbubbles can circulate for a few minutes with the shell accumulating in the liver and spleen, and the gas core exhaled through the lungs [82].

Acoustically active liposomes can have drug-encapsulation efficiency as high as $15 \%$, and drug is released by $1 \mathrm{MHz}$ ultrasound at $2 \mathrm{~W} / \mathrm{cm}^{2}$ for $10 \mathrm{~s}$. The sensitivity of liposomes to ultrasound stimulation could be increased by the inclusion of $4 \%$ diheptanolyphosphatidylcholine (DHPC), which has a high correlation with the loss of gaseous core in the acoustic field. Overall, the current encapsulation and triggered release techniques in liposomes have a high efficiency for great potential in drug delivery.

Initially, there is no release of DOX from either LTSLs or NTSLs. After 2-min incubation, the LTSLs began releasing DOX at a temperature of $39^{\circ} \mathrm{C}(\sim 35 \%$ of payload), more at $42^{\circ} \mathrm{C}(\sim 50 \%)$, and approached $100 \%$ after $12 \mathrm{~min}$. In contrast, the NTSLs did not release any detectable levels of encapsulated DOX at $42^{\circ} \mathrm{C}$ after $12 \mathrm{~min}$. When LTSLs were exposed to pulsed high-intensity focused ultrasound (HIFU), a 3- to 4-fold increase in concentration of DOX in a more rapid manner was found in vivo [83]. 
(a)

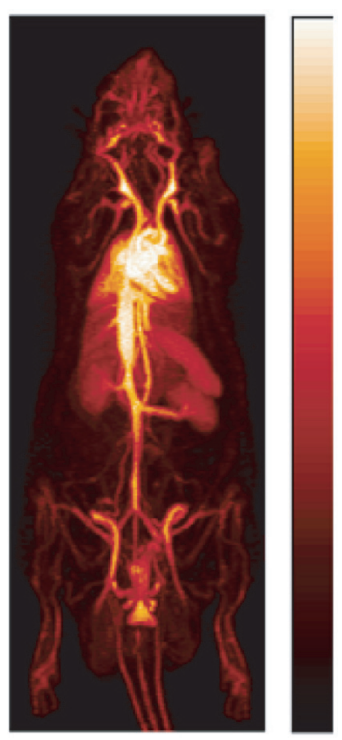

(b)

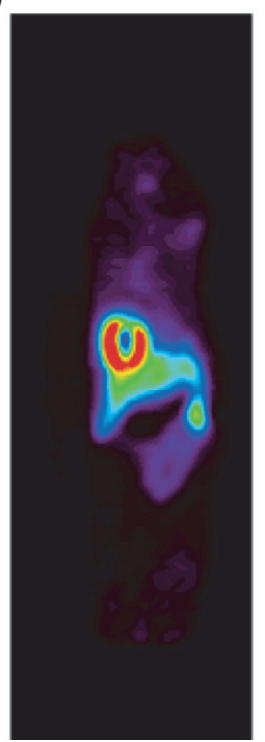

(c)

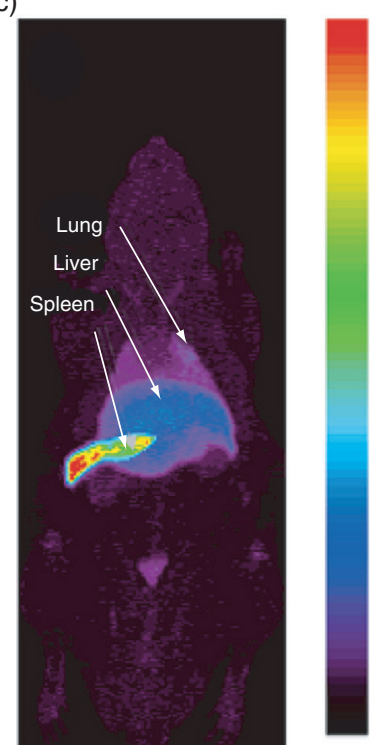

Figure 11. Positron emission tomography (PET) 90-min maximum intensity projection images of (a) long-circulating liposomes, (b) short hearttargeted peptide (Cys-Arg-Pro-Pro-Arg) coated liposomes, and (c) microbubbles in a rat model. Particles were radiolabeled by incorporating $\left[{ }^{18} \mathrm{~F}\right] \mathrm{FDP}$ into the particle shell (used with permission [41]).

\subsection{Micelles}

In the first clinical trial of micellar drug in Japan, DOX was chemically conjugated to PEG-P(Asp) chains and protected from digestion by erythrocytes. This micelle manifested a higher DOX concentration in the solid tumor, but exhibited a significant decrease in cardiotoxicity at the DOX dose of $100 \mathrm{mg} / \mathrm{kg}[48,84]$. In rats receiving DOX-encapsulated micelles, the sonicated tumor grew less than the bilateral tumors, despite large variation among each group, and incomplete tumor regression during the time investigated. In comparison, the tumor volume increased exponentially over time in the negative control group. The slowed tumor growth was due to either increased DOX concentration in the vicinity of the tumor or interaction among the ultrasound, the tumor, and DOX leached from the micelle. Ultrasound promotes the extravasation into the tumor capillaries of any Plurogel drug carriers. The increased permeability of angiogenic vessels and the quantity of DOX from stable micelles improved hepatic colorectal metastases in a mouse model. If defects occur in the cell membrane, ultrasound could enhance the drug uptake to the tumor, irrespective of whether being released from a nearby Plurogel by sonication or having previously diffused out of the Plurogel. Because extensive cell lysis can cause a massive release of lysosomal enzymes and acute inflammation, there should be a compromise between the enhanced drug uptake and cell lysis for the optimal outcome. 
Drug release from micelles could be due to either the diffusion out of micelles or micelle perturbation/degradation by ultrasound. The released drug was quickly reencapsulated in the interval time of sonication pulses so as to minimize the side effects to normal tissues (Figure 12). At pulse duration longer than the threshold, up to $10 \%$ release of DOX is close to that in continuous wave $(\mathrm{CW})$ mode [85]. Pulsed wave is preferred to $\mathrm{CW}$ by virtue of minimizing potential overheating in the clinical practice. Longer pulses and shorter intervals could keep a high concentration of released drug consistently throughout the sonication. The drug release from Pluronic micelles, which has comparable characteristic times of the intracellular drug uptake, increases with the pulse duration, but not the pulse interval. The drug re-encapsulation rate does not depend on the pulse duration. If intervals are long enough, complete re-encapsulation of the released drug occurs.

Optimal stability, low toxicity, and long half-life time of encapsulated DOX were achieved by mixing Pluronic P-105 and polyethylene oxide (PEO)-diacylphospholipid. In addition to the increased intracellular uptake, a uniform distribution of the micelles

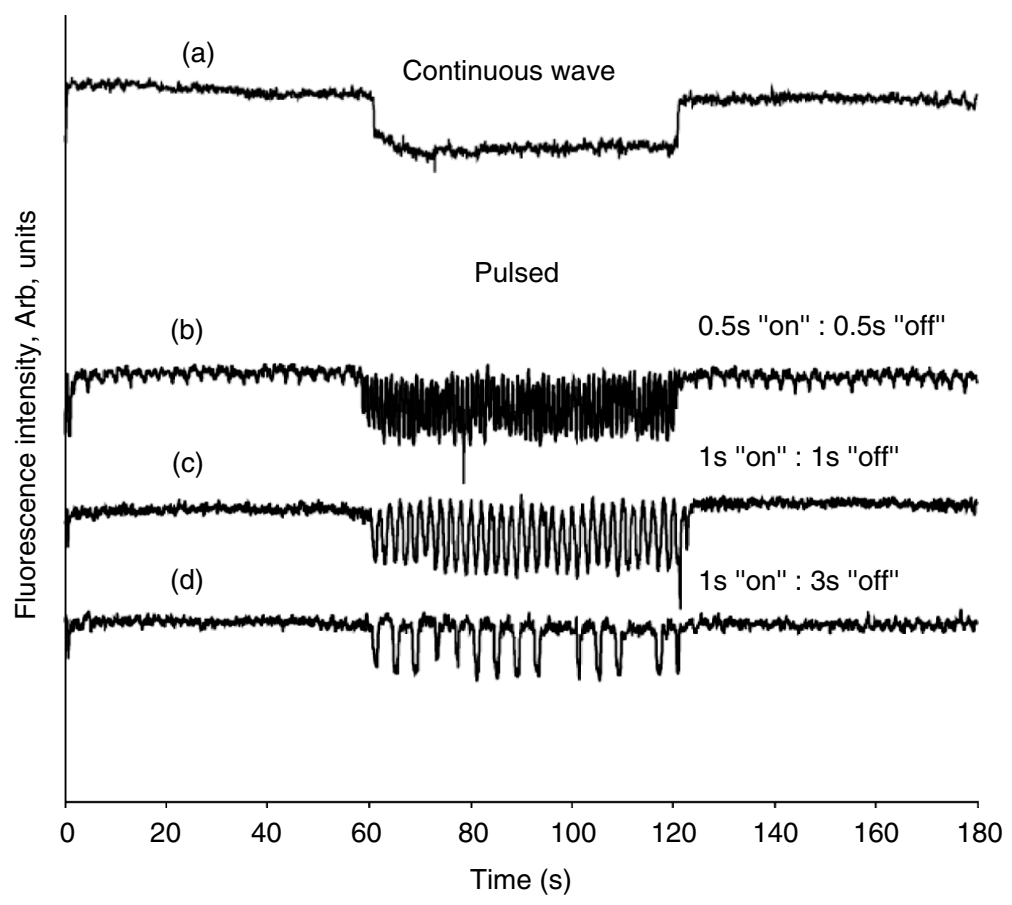

Figure 12. Profile of doxorubicin (DOX) release profiles from $10 \%$ Pluronic micelles under continuous wave $(\mathrm{CW})$ or pulsed $20 \mathrm{kHz}$ ultrasound at an acoustic intensity of $0.058 \mathrm{~W} / \mathrm{cm}^{2}$. The decrease of DOX fluorescence intensity was used to indicate the concentration of DOX in the aqueous medium (used with permission [85]). 
and drug in the tumor volume were found after the sonication via the EPR effect, without molecular targeting (Figure 13). For stabilized Pluronic micelles, the optimal sonication was $4-8 \mathrm{~h}$ after the injection [87]. Despite excellent performance in vitro, Plurogels induced significant cell dehydration in vivo.

Multi-drug resistance (MDR) impedes chemotherapy for metastatic diseases. One of the well-understood mechanisms of MDR is that over-expression of P-glycoprotein (P-gp) enables tumor cells to expel many structurally and functionally unrelated hydrophobic anticancer drugs using the energy of ATP hydrolysis. Most of the P-gp modulating agents, such as verapamil, are cytotoxic, and lack specificity due to the abundance of P-gp. The enhancement of intracellular uptake and cytotoxicity of DOX as well as inhibition of drug efflux produced by ultrasound hyperthermia was far better than that produced by the P-gp modulator. Simultaneously, Pluronic micelles also shielded the drug uptake. These two processes compete with each other, depending on micelle concentration and micelle-drug interaction. Pluronic F-128 at concentration up to $1 \mathrm{wt}$ \% has obtained clinical approval from FDA. Comparative

(a)
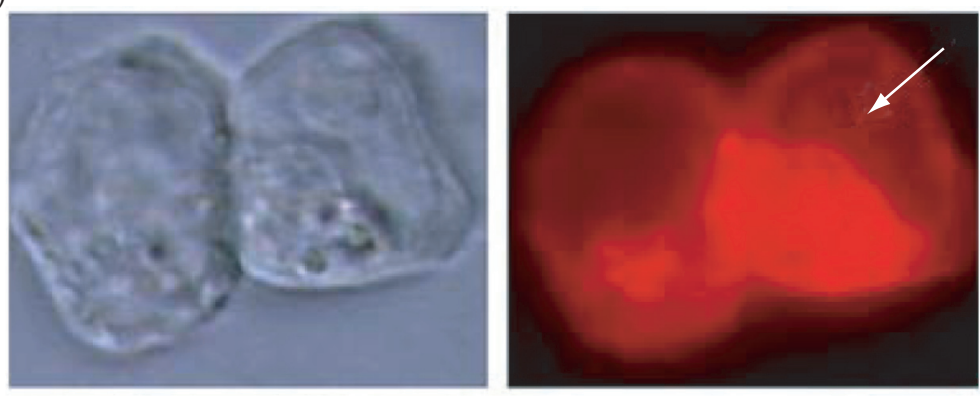

(b)
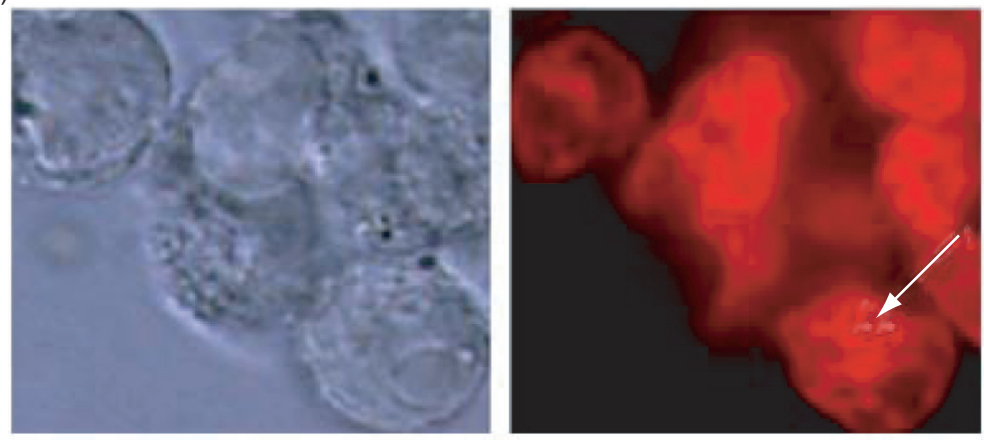

Figure 13. Subcellular trafficking of the Ruboxil $(\mathrm{Rb})(\mathrm{a})$ did not penetrate into the cell nuclei (indicated by arrow), and (b) in 1\% Pluronic P-105 micelles effectively accumulated in the cell nuclei of the multi-drug resistant ovarian carcinoma cells (used with permission [86]). 
studies of chemotherapy with and without the use of ultrasound ( 20 and $70 \mathrm{kHz}$ ) have been carried out in mice bearing highly MDR human colon KM20 tumors [79]. Sonication alone does not produce tumor regression or affect the tumor growth rate significantly. Without anti-cancer drug, sonication in combination with the nanoparticles resulted in a temporal decrease of tumor volume. However, the tumor regression occurred several days later, suggesting that it was due to mechanical damage to tumor blood vessels by bubble cavitation (SC and IC). There was a statistical difference between sonicated and control tumors $(p<0.05)$ for up to 2 months in mice. No metastatic tumors were observed in the internal organs.

Drug release from micelles decreases with driving frequency (i.e., from $20 \mathrm{kHz}$ to $3 \mathrm{MHz}$ ) and increases linearly with acoustic intensity for the stabilized Pluronic micelles. However, there is no acoustic intensity threshold in drug release from micelles, suggesting that IC is not the sole mechanism involved. Decreased drug efflux and increased nuclear transport resulted in a dramatic sensitization of the MDR cells in the presence of Pluronic micelles, more than that on non-cancerous cells. Despite decreased intracellular drug uptake by Pluronic micelles due to the induced deactivation of drug efflux pumps by the decreased energy in the MDR cells, the high-growth inhibition was almost the same for micellar-encapsulated and free drug, possibly because of the cytostatic action of Pluronic micelles overcoming the cytotoxic action of the drug. Such an inhibition effect on MDR cell growth was more significant than that on drug-sensitive cells. The presence of Pluronic unimers rather than that of micelles at the absence of drugs could enhance both cytostatic and cytotoxic actions. All in all, localized and controlled drug delivery to drug-sensitive and MDR tumors by the synergy of drug, micelle, and ultrasound techniques that can effectively deliver drugs is feasible, and will be further evaluated in the coming in vivo experiments.

\subsection{Gene Expression}

Microbubbles (i.e., Levovist ${ }^{\circledR}$, Albunex ${ }^{\circledR}$, and Optison ${ }^{\circledR}$ ) with ultrasound can have up to 6-fold increase in the beta-Gal gene transfection to vascular smooth muscle cells (VSMCs) in comparison to the mixture of genes and VSMCs. If adenovirus genes are loaded on the lipid shell of microbubbles, a 10-fold increase in a mouse heart model can be achieved. Delivery to a specific site can be aided using the targeted microbubbles. Bubble cavitation (SC and IC) leads to a fusion between viral vectors and cell membranes rather than a forced deposition of intact virus particles inside the cytoplasm. In this way, the virus can unpack and easily proceed to the nucleus. Also remarkable was the rather uniform gene transfer in the cardiac tissue, probably due to the ability of adeno-associated virus (AAV) serotypes to cross blood vessel barriers and spread through the extracellular matrix [22]. Moreover, adverse immune responses can be drastically reduced, as the virus is shielded from the immune system. A transfection efficiency of 50\% was comparable to that achieved using lipofection. As a physical method, ultrasound does not have limitations in plasmid DNA (pDNA) uptake into cells by lipofection, such as the net charge of the cationic lipids, DNA complexes and concentrations, the $\mathrm{pH}$, and concentration of electrolytes. The optimization of different cationic lipids used for each lipofection experiment leads to 
inconsistent results [55]. Although relatively large amounts of pDNA are needed for competitive transfection rate in sonication, the in vivo site specificity generally outweighs this small disadvantage [88].

It was observed that macromolecules with different MWs have similar penetration capability through plasma membrane [80]. In MCF7 cells, the overall difference between the delivery rates of $10 \mathrm{kDa}$ and $2000 \mathrm{kDa}$ dextrans was about $20 \%$. However, confocal microscopy revealed that intracellular distribution of the delivered macromolecules depended upon their size. Macromolecules of $2000 \mathrm{kDa}$ did not penetrate through the nuclear membrane, while $10 \mathrm{kDa}$ macromolecules were uniformly distributed within the cells. Macromolecules of MW $70 \mathrm{kDa}$ can penetrate through the nuclear membrane; however, nuclear fluorescence of FITC-dextran is weaker than cytoplasmic, suggesting that $70 \mathrm{kDa}$ is close to a threshold of nucleus penetration. At the same sonication conditions, SK-BR-3 human breast carcinoma cells demonstrated only $3.5 \%$ of dead rate, while the value for HeLa human cervix epithelial adenocarcinoma cells was about 40\% [80]. Moreover, KM20 human colorectal carcinoma cells demonstrated more than $70 \%$ loading with macromolecular drugs, and only $5 \%$ of dead cells, but $10 \%$ cells expressing EGFP $24 \mathrm{~h}$ after sonication [80]. Shock waves have been shown to increase cellular uptake of both small (i.e., adriamycin and fluorescein) and macromolecules (i.e., fluorescein-labeled dextrans, ribosomeinactivating proteins gelonin, and saporin). Utilizing $1 \mathrm{MHz}$ ultrasound at a spatial average peak positive pressure of $0.41 \mathrm{MPa}\left(\sim 10 \mathrm{~W} / \mathrm{cm}^{2}\right)$, a better transfection of $50 \%$ in the living cells can be achieved, similar to the outcome of lipofection. Therefore, ultrasound-induced gene delivery may be more or less suitable for different cell types due to the natural variation, and further optimization of exposure conditions may improve transfection.

\subsection{Sonodynamic Therapy}

SDT can enhance the toxicity of certain compounds (i.e., haematoporphyrin, pheophorbide, erythrosin B, and ATX-70). For example, $25 \mathrm{kHz}$ ultrasound caused lethal damage to leukemia cells in the presence of merocyanine 540 in vitro. Hematoporphyrin, the most common photodynamic sensitizer, with $1.92 \mathrm{MHz}$ ultrasound at intensities of 1.27 and $3.18 \mathrm{~W} / \mathrm{cm}^{2}$ enhanced the death rate of mouse sarcoma and rat ascites 130 tumor cells from 30\% to $99 \%$, and from 50\% to $95 \%$, respectively. The inhibitory effects of hematoporphyrin derivatives on the tumor cell growth were confirmed by the cell's morphological changes, cytochrome C oxidase activity, and degradation of DNA. No cytotoxicity and structural modification of hematoporphyrin derivative in sonication was observed on in vitro human colorectal adenocarcinoma cells (HT-29) and Chinese hamster ovary cells. Sixty seconds of low frequency ultrasound $(270 \mathrm{kHz})$ at intensities of $0.15,0.3$, and $0.45 \mathrm{~W} / \mathrm{cm}^{2}$ and Photofrin II (commercial and purified hematoporphyrin derivative) decreased the survival of HL-60 cells, $92.9 \pm 1.5 \%$ vs. $49.6 \pm 5.1 \%$ (without vs. with Photofrin), $82.3 \pm 2.2 \%$ vs. $34.5 \pm 3.1 \%$, and $77.0 \pm 7.2 \%$ vs. $27.4 \pm 3.0 \%$, respectively. In an SDT experiment $\left(450 \mathrm{kHz}\right.$ at an intensity of $\left.0.3-0.5 \mathrm{~W} / \mathrm{cm}^{2}\right)$, the survival rate of MT-2 cells was inversely proportional to the amount of Photofrin. The corresponding values of 
peripheral mononuclear cells in the blood of acute-type adult $\mathrm{T}$ cell leukemia patients after ultrasound exposure $\left(0.3 \mathrm{~W} / \mathrm{cm}^{2}, 60 \mathrm{~s}\right)$ alone, and together with $100 \mu \mathrm{g} / \mathrm{ml}$ of Photofrin were $69.4 \pm 22.5 \%$ and $30.0 \pm 23.0 \%$, respectively. In contrast, there was no significant difference of survival rates of normal human peripheral mononuclear cells between ultrasound-treated groups with and without Photofrin. Therefore, SDT may be an effective and extracorporeal blood treatment modality for acute-type adult $\mathrm{T}$ cell leukemia patients [89].

\section{DISCUSSION AND FUTURE DIRECTIONS}

\subsection{Clinical Translation}

The ultrasound-mediated drug delivery uses the same microbubbles or liposomes for diagnosis and therapy at the tumor-specific region. Each component of this modality fulfills its own function: drug vehicles target to specific tumors precisely; ultrasound triggers the drug release in a temporally controlled manner, enhances drug diffusion through the vessel wall for a more uniform distribution in the tumor, and perturbs cell membranes for the intracellular drug uptake. Therefore, it is progressing toward a feasible approach for solid tumors (i.e., breast, colon, ovaries, uterus, and larynx) treatment with the following important and attractive aspects [65]: (a) Microbubbles or echogenic agents have already been used in clinical sonography. (b) It is possible to selectively target drug delivery to the sonicated area at the deeply seated tumor using image guidance (i.e., ultrasound, computed tomography (CT), magnetic resonance imaging (MRI), or PET) with a high positioning accuracy, which may be beneficial in noninvasive treatment of a localized tumor. (c) Ultrasound exposure is a noninvasive and extracorporeal method in comparison to radiofrequency and microwave ablation, which require insertion of interstitial needle or antenna. (d) Delivery of various agents (i.e., small molecules, DNA, and nanoparticles) to a variety of tissues with satisfactory therapeutic efficacy by mechanical, thermal effect, or combination is possible. (e) Drug release was rapid at the sonicated region only, and such target specificity is much better than current chemotherapy. (f) It could also be combined with other adjuvant therapies to avoid or reduce metastasis. However, the drawback is relatively small volumes (a few millimeters in the lateral by about 1 centimeter in the axial directions). In clinical treatment, the HIFU focus is required to be scanned throughout the whole target volume, which may take hours depending on the target size and treatment planning. Although extensive studies have been carried out both in vitro and in vivo with promising results, little significant progress has been made in the clinical practice. During the last decade, combined diagnostic and therapeutic ultrasound (i.e., HIFU) has been tested in clinical trials and has attracted growing interest, and concomitant instrumentation is available on the market, such as ExAblate (InSightec, Israel), Achieva (Philips, Briarcliff, NY, USA), JC (Chongqing Haifu (HIFU) Technology, Chongqing, China), and Theraclion (Malakoff, France). Ultrasound-mediated drug delivery may be rapidly applied to humans with only changes of operation parameters in existing HIFU devices. However, this technology is still in its infancy, and that road ahead presents a number of exciting opportunities as well as pitfalls that will need to be circumvented. 


\subsection{Ultrasound Parameter Optimization}

A major challenge is to understand the mechanisms involved in this technology in order to optimize the ultrasound parameters. For example, for effective molecular internalization facilitated by the increased cell permeability, the plasmid should be close to the target because the cells return almost immediately to their initially impermeable state after sonication. Therefore, the transient pore opening limits the efficiency and quantity of delivery. If the IC-induced pores in the cell membrane are too large to reseal quickly, the cell may lose vital cytoplasmic compound. The primary cause for cell death of sonoporation is likely to be a mechanical effect, resulting from shear stresses to the cell membranes by violent IC or microstreaming, which was supported by the observation that a large number of cells disappeared shortly after exposure (approximately ten minutes), but before any slow biochemical processes (i.e., oncosis and apoptosis). Therefore, there should be a tradeoff between these two phenomena for high rate of drug penetration through the membrane into cell cytoplasm and cell nucleus, as well as sufficient cell viability. The dominant effects need be monitored throughout the whole process of drug delivery. Bubble cavitation (SC and IC) activities need to be detected as the dose of the mechanical effect, and then used as feedback in a closed-loop control system for effective and consistent outcome. Similarly, the temperature elevation also needs to be measured when temperature-sensitive drug vehicles are used.

\subsection{Drug/Gene Vehicle Optimization}

In order to improve the performance of ultrasound-mediated drug/gene delivery, efforts are focused to (a) enhance the stability of the vesicle, (b) increase drug/gene loading, and (c) improve targeting [41, 42]. Air sensitivity in liposome can be increased by adding short-chain lipid (i.e., DHPC) without entrapment of air or calcein marker. Although DHPC can destabilize bilayers by lowering their lysis tension, such a phenomenon is not the critical factor for lytic events. A general drawback of microbubbles as drug carriers is the rather small space available for agents loading. Targeting ligands may prevent an efficient loading on the shell with agents, especially via electrostatic interactions between the drug and the shell. Drug release is restricted to the sonicated areas with low acoustic intensity, and therefore allowing a low drug dose. Their relatively short in vivo half-life limits the therapeutic irradiation. Because microbubbles have a broad size distribution, they will not respond evenly to a given ultrasound frequency. Small sub-microbubbles might be able to extravasate more easily, especially in the tumor vasculature (380-780 nm of pore size), but can be rapidly taken up by RES [90-92]. In contrast, larger microbubbles can carry a higher drug payload and are more easily destroyed at relatively low acoustic intensities [22].

An important advantage of ELPs over other thermo-sensitive carriers is that drug accumulates because of the phase transition of the ELP rather than triggered drug release [40]. Therefore, a concentration gradient is not required to drive accumulation, and ELP-drug injected at a low concentration aggregates in the heated tumor. Second, in comparison to antibody and other affinity targeting approaches, the aggregation of ELP-radionuclide conjugates can directly target the heated tumor microvasculature, and 
circumvent the barriers associated with extravasation. Third, the clinical implementation does not require any concomitant development in hyperthermia. Finally, the aggregated ELP will resolubilize due to reversion of the phase transition after cessation of hyperthermia.

Electroporation, a popular experimental procedure in gene transfection, requires manipulating electric fields by setting and positioning two electrodes to cells. In comparison, sonoporation requires a much simpler setting especially in vivo [71]. Bubble cavitation (SC and IC) may damage endothelial cells in both arteries and capillaries, through which pDNA can be transfected effectively into cardiomyocytes. Due to its small size, siRNA could pass through the coronary arteries and arterioles, resulting in efficient transduction from the bloodstream into arterial/arteriolar walls including the smooth muscle cells. Although pDNA permeates endothelium slower, its transduction is predominant at the capillary bed with intense IC. The efficacy of gene transduction depends on the types and properties of the cells, because neuroectoderm and limb ectoderm cells tend to express exogenous genes after a relatively short period of sonication. As part of such differences of gene transduction efficacy, it will be important to regulate the state of diffusion or the retention of injected solution to the target. A higher concentration of injected DNA may increase the viscosity of the DNA-microbubble mixture, and lead to the failure of increment of gene transduction efficiency in neural tubes.

The poor water solubility of some sonosensitizers, mainly due to the physicalchemical properties of hypocrellin, leads to the easy aggregation in aqueous media and limits their clinical applications. As a result, their concentration in a specific target may not be sufficient for the therapeutic requirement. One of the solutions is nanotechnology in medicine, that is able to manipulate molecules and supramolecular structures to produce programmed functions. Encapsulated sonosensitier by hydrophobic nanoparticles has been shown to enhance the circulation time and prevent uptake by RES. The enormous surface area of the nanoparticles can be modified for an array of functionalities with diverse chemical or biochemical properties. In addition, presence of nanoparticles of the appropriate size and amount in the liquid could provide nucleation sites, decrease the cavitation threshold, and increase the liquid temperature in the sonication because of their surface roughness. Gold nanoparticles are highly attractive for their low toxicity, good uptake by mammalian cells, and antiangiogenetic properties. In the presence of gold nanoparticles, the relaxation time of protoporphyrin IX increases, favoring efficient production of ROS [93]. Inorganic nanoparticles, such as titanium dioxide $\left(\mathrm{TiO}_{2}\right)$, have a strong interaction with light for SDT. Although $\mathrm{TiO}_{2}$ cannot be used as a SDT drug alone because of its insufficient selectivity and low efficiency in cancer cells, it can work as an effective sonocatalyzer in the treatment of bladder cancer and in glioma cell lines [94]. Since the long-term side effects of inorganic nanoparticle accumulation are still unknown, luminescent silica nanoparticles that decompose in the aqueous medium in hours may be utilized as a sonosensitizer.

\subsection{Bioeffects of the Cell Membrane}

Disruption of the cell membrane is one of the mechanisms of ultrasound-mediated drug delivery. Sonoporated cells may respond to the disrupted intracellular equilibrium by up-regulating cytoplasmic signals related to apoptosis and cell-cycle 
arrest. Ultrasound can also generate calcium transients (i.e., changes of intracellular calcium concentration, oscillations, and spatial waves of $\left.\left[\mathrm{Ca}^{2+}\right]_{\mathrm{i}}\right) . \mathrm{Ca}^{2+}$ is an important messenger involved in regulating many cellular processes and functions, such as gene transcription, cell proliferation, fertilization, metabolism, cell migration, wound response, and phagocytosis [95]. Bubble cavitation (SC and IC) produces an intracellular increase of $\left[\mathrm{Ca}^{2+}\right]_{i}$ hundreds of $\mu \mathrm{m}$ away in a few seconds. $\left[\mathrm{Ca}^{2+}\right]_{\mathrm{i}}$ activities affect cell-cell contact, electrical resistance, ZO-1 tight junction protein migration from intracellular sites to the plasma membrane, and tight junction assembly in epithelial and endothelial cells [96]. Meanwhile, mitosis of the cell cycle is halted through down-regulation of the Cdc-2 protein. Apoptosis may be triggered through mitochondria and promote cytochrome-c into the cytoplasm via poly-ADPribose polymerase (PARP) cleavage by the activation of the caspase-3 protein. Resealing of the cell membrane is not a simple self-reunion procedure, since the scattered patches on the same size order as membrane pores were observed on the resealed cells [97]. It is hypothesized that an exocytotic patching mechanism may be involved so that cytoplasmic vesicle will first be delivered to the sonoporation site and then fused together with the membrane, which is similar to the $\left[\mathrm{Ca}^{2+}\right]_{\mathrm{i}}$ influx into porous cells.

Another barrier of interest is DNA trafficking to the nucleus. Ultrasound can deliver macromolecules into the cytosol, but does not promote their transport into the nucleus [98]. Microscopy illustrated that intracellular DNA was excluded from the nucleus, which is consistent in the other gene therapy methods. Therefore, understanding the intracellular pDNA trafficking would optimize the ultrasound-mediated gene transfection.

\subsection{Other Applications}

The blood-brain barrier (BBB) consists of tight junctions around all capillaries in the central nervous system (CNS), which separates circulating blood from the brain extracellular fluid. Because of the presence of multiple endogenous transporters, BBB allows a selective entry of small-size nutrients and minerals across it, but restricts the entry of large substances, such as neuropharmaceutical agents for CNS disorders (i.e., Alzheimer's disease, dementia, Parkinson's disease, mood disorder, AIDS, viral and bacterial meningitis), for maintaining the internal milieu of the brain. Therefore, several novel approaches, such as nanoparticles, liposomes, antibody-mediated delivery approaches and application of genomics [99] are in development for brain target. Recently, it was found that transcranial focused ultrasound can transiently permeabilize the BBB, increasing passive diffusion in the presence of microbubbles [100-102]. Subsequent application of an external magnetic field then actively enhances localization of a chemotherapeutic agent monitored by MRI. Combining these techniques significantly improved the delivery of 1,3-bis(2-chloroethyl)-1-nitrosourea (BCNU) to rodent gliomas for suppressing tumor progression without damage to normal tissue as indicated in MRI and histology. This novel technique promises a more effective and tolerable means of tumor therapy, with lower therapeutic doses and concurrent clinical monitoring [103, 104]. Furthermore, it can also be used for the treatment of thrombus, restenosis, and angiogenesis. 
The use of ultrasound for the delivery of drugs to, or through, the skin is commonly known as sonophoresis or phonophoresis. The use of high-frequency therapeutic ultrasound ( $\geq 0.7 \mathrm{MHz}$ ) for sonophoresis (viz., high-frequency sonophoresis, HFS) occurred as early as the 1950s, while low-frequency sonophoresis (LFS, 20-100 kHz) has been investigated during the past two decades. Although HFS and LFS are similar to the concept of utilizing ultrasound to increase the skin penetration, the underlying mechanisms are different (bubble cavitation (SC and IC) and mechanical effect dominating LFS and HFS, respectively). Aside from the benefits of avoiding the hepatic first-pass effect, and higher patient compliance, the additional advantages include strict control of transdermal penetration rate, rapid termination, low risk of infection, less anxiety-provoking or painful than injection, and not immunologically sensitizing, despite potential disadvantages of minor tingling, irritating and burning. Both HFS and LFS improved current methods of local, regional, and systemic drug delivery or even vaccine in the future $[105,106]$.

\subsection{Summary}

Overall, the research and application in ultrasound-mediated drug/gene delivery have advanced tremendously during the past decade, and are expected to continue. Future technological growth may be concentrated on, but not limited to, the following aspects: (a) the understanding of the interaction of ultrasound with acoustically activated drug vehicles and the subsequent bioeffects; (b) the development of drug vehicles for effective penetration through endothelial junctions that are typically damaged in systemic inflammation or tumor vasculature to target specific receptors rather than on the vascular endothelium (high specificity), more effective drug/gene loading, and sufficient circulating lifespan; (c) response of cancer/tumor cells to ultrasound (sonoporation) and drug (apopotosis); (d) optimization of ultrasound parameters for drug diagnosis and release in different organs with tradeoff between cell viability and drug/gene transduction; (e) in situ real-time control by detecting the IC, temperature, or drug concentration; and (f) large animal experiments and translation into clinical trials.

\section{CONCLUSIONS}

The most common cancer treatment, chemotherapy, is often limited by its cytotoxic effects on normal tissues, particularly under high doses. Therefore, it is highly desirable to reduce the dosage or frequency of administration by enhancing the effectiveness of drugs to the specific target. The application of ultrasound in delivery of several therapeutic classes (i.e., chemotherapeutic, thrombolytic, and DNA-based drugs) acting via bubble cavitation (SC and IC), radiation forces, and/or heat has recently gained impetus. The advantages of this novel technology include noninvasiveness, low cost, easy operation, good focusing and penetration inside the body, and no radiation. Imaging methods (i.e., sonography and MRI) may be particularly helpful in defining the target, determining local drug concentration, and evaluating efficacy and temperature. Recent successes suggest that ultrasound may be a valuable therapeutic tool for drug delivery by lowering the administration dosage of an anti-cancer drug. However, this technology is still in its infancy. Progression to clinical implementation will depend on the success of resolving several key issues. The core is the appropriate design of drug vehicles and assemblies, such as microbubble, liposomes, and micelles, capable of carrying sufficient 
payload, yet maintaining specific response to acoustic energy. Conjugating tumor- and disease-specific antigens or binding ligands to the surface of drug vehicles can improve their specificity for targets without compromising either echogenicity or payload capacity. Prolonged circulation and minimal removal by RES are also required. Only by overcoming these obstacles can ultrasound translate from bench to bedside. An increasing body of knowledge has been acquired on the interaction between ultrasound energy with tissues, therapeutic agents, and drug carriers for enhanced therapeutic efficacy and efficiency. With advances in the transducer and sonography technologies, a variety of ultrasound-based therapeutic applications have been and will continue to be developed. For technical improvement and translation from one tissue type to another, an in-depth understanding of the ultrasound mechanisms and physical characteristics of the tissues, both in vitro and in vivo data, and mathematical models to optimize the treatment protocols will be required. It must be noted that this technique has mainly been tested in-vitro or in small animal studies to date. There is a great need for large animal and human studies. Moreover, ultrasound-mediated gene delivery seems to outweigh drug delivery in certain applications, such as cardiovascular disease treatment. All in all, ultrasound-mediated drug/gene delivery is a promising technology that attracts the interests of both scientific research and medical applications.

\section{CONFLICT OF INTEREST}

The author indicated no potential conflicts of interest.

\section{REFERENCES}

[1] Howlader N, Noone AM, Krapchom M, Neyman N, Aminou R, Waldron W, Altekruse SF, Kosary CL, Ruhl J, Tatalovich Z, Cho H, Mariotto A, Eisner MP, DLewis DR, Chen HS, Feuer EJ, Cronin KA and Edwards BK. SEER cancer statistics review. National Cancer Institute, 2011, 1975-2008.

[2] Curti BD. Physical barriers to drug delivery in tumors, in: B.A. Chabner (ed). Cancer Chemotherapy and Biotherapy, 1996, 709-719.

[3] Jain RK. Normalization of tumor vasculature: an emerging concept in antiangiogenic therapy. Science, 2005, 307: 58-62.

[4] Boucher Y, Baxter LT and Jain RK. Interstitial pressure gradients in tissue-isolated and subcutaneous tumors: implications for therapy. Cancer Res., 1990, 50: 4478-4484.

[5] McKee TD, Grandi P, Mok W, Alexandrakis G, Insin N, Zimmer JP, Bawendi M, Boucher Y, Breakefield XO and Jain RK. Degradation of fibrillar collagen in a human melanoma xenograft improves the efficacy of an oncolytic herpes simplex virus vector. Cancer Res., 2006, 6: 2509-2513.

[6] Awada A and Piccart M. Strategies offering protection from the toxic effects of anticancer treatments with a focus on chemoprotective agents. Curr. Opin. Oncol., 2000, 12: 289-296.

[7] Floyd JD, Nguyen DT, Lobins RL, Bashir Q, Doll DC and Perry MC. Cardiotoxicity of cancer therapy. J. Clin. Oncol., 2005, 23: 7685-7696.

[8] Briggers I, Dubernet C and Couvreur P. Nanoparticles in cancer therapy and diagnosis. Advanced Drug Delivery Reviews, 2012, 54: 631-651.

[9] Hernot S and Klibanov AL. Microbubbles in ultrasound-triggered drug and gene delivery. Advanced Drug Delivery Reviews, 2008, 60: 1153-1166.

[10] Kremkau FW, Kaufmann JS, Walke MM, Burch PG and Spurr CL. Ultrasonic enhancement of nitrogen mustard cytotoxicity in mouse leukemia. Cancer, 1976, 37: 1643-1647.

[11] ter Haar G. Ultrasound mediated drug delivery: A 21st century phoenix? International journal of hyperthermia: the official journal of European Society for Hyperthermic Oncology, North American Hyperthermia Group, 2012, 28(4): 279. 
[12] Postema M and Gilja OH. Ultrasound-directed drug delivery. Current pharmaceutical biotechnology, 2007, 8(6): 355-361.

[13] Tachibana K and Tachibana S. The use of ultrasound for drug delivery. Echocardiography, 2003, 18(4): 323-328.

[14] Shortencarier MJ, Dayton PA, Bloch SH, Schumann PA, Matsunaga TO and Ferrara KW. A method for radiation-force localized drug delivery using gas-filled liposomes. IEEE transactions on Ultrasonics, Ferroelectrics and Frequency Control, 2004, 51: 822-831.

[15] Cosgrove D. Ultrasound contrast agents: an overview. European Journal of Radiology, 2006, 60(3): 324-330.

[16] Dolan MS, Gala SS, Dodla S, Abdelmoneim SS, Xie F, Cloutier D, Bierig M, Mulvagh SL, Porter TR and Labovitz AJ. Safety and efficacy of commercially available ultrasound contrast agents for rest and stress echocardiography: a multicenter experience. Journal of the American College of Cardiology, 2009, 53(1): 32-38.

[17] Ferrara K, Pollard R and Borden M. Ultrasound microbubble contrast agents: fundamentals and applications to gene and drug delivery. Annual Review of Biomedical Engineering, 2007, 9: 415-447.

[18] Klibanov AL. Microbubble contrast agents: targeted ultraound imaging and ultrasound-assisted drugdelivery applications. Investigative Radiology, 2006, 41(3): 354-362.

[19] Unger EC, Matsunaga TO, McCreery T, Schumann P, Sweitzer R and Quigley R. Therapeutic applications of microbubbles. European Journal of Radiology, 2002, 42: 160-168.

[20] Unger EC, Hersh E, Vannan M, Matsunaga TO and McCreery T. Local drug and gene delivery through microbubbles. Progress in Cardiovascular Diseases, 2001, 44(1): 45-54.

[21] Unger EC, McCreery TP, Sweitzer RH, Caldwell VE and Wu Y. Acoustically active lipospheres containing paclitaxel: a new therapeutic ultrasound contrast agent. Investigative Radiology, 1998, 33(12): 886.

[22] Lentacker I, De Smedt SC and Sanders NN. Drug loaded microbubble design for ultrasound triggered delivery. Soft Matter, 2009, 5: 2161-2170.

[23] Klibanov AL. Targeted delivery of gas-filled microspheres, contrast agents for ultrasound imaging. Advanced Drug Delivery Reviews, 1999, 37: 139-157.

[24] Villanueva FS, Jankowski RJ, Klibanov S, Pina ML, Alber SM, Watkins SC, Brandenburger GH and W.R. W. Microbubbles targeted to intercellular adhesion molecule-1 bind to activated coronary artery endothelial cells. Circulation, 1998, 98: 1-5.

[25] Weller GE, Lu E, Csikari MM, Klibanov AL, Fischer D, Wagner WR and Villanueva FS. Ultrasound imaging of acute cardiac transplant rejection with microbubbles targeted to intercellular adhesion molecule-1. Circulation, 2003, 108: 218-224.

[26] Weller GE, Wong MK, Modzelewski RA, Lu E, Klibanov AL, Wagner WR and Villanueva FS. Ultrasonic imaging of tumor angiogenesis using contrast microbubbles targeted via the tumor-binding peptide arginine-arginine-leucine. Cancer Res., 2005, 65: 533-539.

[27] Hauff P, Reinhardt M, Briel A, Debus N and Schirner M. Molecular targeting of lymph nodes with Lselectin lignad-specific us contrast agent: a feasibility study in mice and dogs. Radiology, 2004, 231: $667-673$.

[28] Schumann PA, Christiansen JP, Quigley RM, McCreery TP, Sweitzer RH, Unger EC, Lindner JR and Matsunaga TO. Targeted-microbubble binding selectively to GPIIb IIIa receptors of platelet thrombi. Invest. Radiol., 2002, 37: 587-593.

[29] Weller GE, Villanueva FS, Tom EM and Wagner WR. Targeted ultrasound contrast agents: in vitro assessment of endothelial dysfunction and multi-targeting to ICAM-1 and sialyl Lewis. Biotechnol. Bioeng., 2005, 92: 780-788.

[30] Borden MA, Sarantos MR, Stieger SM, Simon SI, Ferrara KW and Dayton PA. Ultrasound radiation force modulates ligand availability on targeted contrast agents. Molecular Imaging, 2006, 5: 139-147.

[31] Rapoport NY, Kennedy AM, Shea JE, Scaife CL and Nam K-H. Controlled and targeted tumor chemotherapy by ultrasound-activated nanoemulsions/microbubbles. J. Controlled Release, 2009, 138: 268-276. 
[32] Campbell R. Tumor physiology and delivery of nanopharmaceuticals. Ant Canc. Agents Med. Chem., 2006, 6(6): 503-512.

[33] Reznik N, Williams R and Burns PN. Investigation of vaporized submicron perfluorocarbon droplets as an ultrasound contrast agent. Ultrasound in Medicine and Bilogy, 2011, 37(8): 1271-1279.

[34] Zhang $\mathrm{P}$ and Porter T. An in vitro of a phase-shift nanoemulsion: A potential nucleation agent for bubble-enhanced HIFU tumor ablation. Ultrasound in Medicine \& Biology, 2010, 36(11): 1856-1866.

[35] Huang SL. Liposomes in ultrasonic drug and gene delivery. Advanced Drug Delivery Reviews, 2008, 60(10): 1167-1176.

[36] Schroeder A, Kost J and Barenholz y. Ultrasound, liposomes, and drug delivery: principles for using ultrasound to control the release of drugs from liposomes. Chemistry and Physics of Lipids, 2009, 162: 1-16.

[37] Allen TM. Liposomes: opportunities in drug delivery. Drugs, 1997, 54: 8-14.

[38] Ranson MR, Carmichael J, O'Byrne K, Stewart S, Smith D and Hwell A. Treatment of advanced breast cancer with sterically stabilized liposomal doxorubicin: results of a multicenter phase II trial. $J$. Clin. Oncol., 1997, 15: 3185-3191.

[39] Gasselhuber A, Dreher MR, Partanen A, Yarmolenko PS, Woods D, Wood BJ and Haemmerich D. Targeted drug delivery by high intensity focused ultrasound mediated hyperthermia combined with temperature-sensitive liposomes: Computational modelling and preliminary in vivo validation. International Journal of Hyperthermia, 2012, 28(4): 337-348.

[40] Chilkoti A, Dreher MR, Meyer DE and Raucher D. Targeted drug delivery by thermally responsive polymers. Advanced Drug Delivery Reviews, 2002, 54: 613-630.

[41] Ferrara KW, M.A. B and Zhang H. Lipid-shelled vehicles: engineering for ultrasound molecular imaging and drug delivery. Accounts of Chemical Research, 2009, 42(7): 881-892.

[42] Huang SL and MacDonald RC. Acoustically active liposomes for drug encapsulation and ultrasoundtriggered release. Biochimica et Biophysica Acta, 2004, 1665: 134-141.

[43] Kheirolomoom A, Dayton PA, Lum AFH, Little E, Paoli EE, Zheng HR and Ferrara KW. Acousticallyactive microbubbles conjugated to liposomes: characterization of a proposed drug delivery vehicle. Journal of Controlled Release, 2007, 118: 275-284.

[44] Endo-Takahashi Y, Negishi Y, Kato Y, Suzuki R, Maruyama K and Aramaki Y. Efficient siRNA delivery using novel siRNA-loaded bubble liposomes and ultrasound. Int. J. Pharm. , 2012, 422(1-2): 504-509.

[45] Negishi Y, Endo-Takahashi Y, Matsuki Y, Kato Y, Takagi N, Suzuki R, Maruyama K and Aramaki Y. Systemic delivery systems of angiogenic gene by novel bubble liposomes containing cationic lipid and ultrasound exposure. Mol. Pharm., 2012, 9(6): 1834-1840.

[46] Lum AFH, M.A. B, Dayton PA, Kruse DE, Simon SI and Ferrara KW. Ultrasound radiation force enables targeted deposition of model drug carriers loaded on microbubbles. J. Controlled Release, 2006, 111: 128-134.

[47] Rapoport N. Combined cancer therapy by micellar-encapsulated drug and ultrasound. International Journal of Pharmaceutics, 2004, 277: 155-162.

[48] Rapoport N. Physical stimuli-responsive polymeric micelles for anti-cancer drug delivery. Progress in Polymer Science, 2007, 32: 962-990.

[49] Marin A, Sun H, Husseini GA, Pitt WG, Christensen DA and Rapoport NY. Drug delivery in pluronic micelles: effect of high-frequency ultrasound on drug release from micelles and intracellular uptake. Journal of Controlled Release, 2002, 84(1): 39-47.

[50] Wan CPL, Jackson JK, Pirmoradi FN, Chiao M and Burt HM. Increased accumulation and retention of micellar paclitaxel in drug-sensitive and P-Glycoprotein-expressing cell lines following ultrasound exposure. Ultrasound in medicine \& biology, 2012, 38(5): 736-744.

[51] Rapoport N. Ultrasound-mediated micellar drug delivery. International Journal of Hyperthermia, 2012, 28(4): 374-385. 
[52] Dijkmans PA, Juffermans LJ, Musters RJ, van Wamel A, ten Cate FJ, van Gilst W, Visser CA, de Jong $\mathrm{N}$ and Kamp O. Microbubbles and ultrasound: from diagnosis to therapy. European Journal of Echocardiography, 2004, 5: 245-256.

[53] ter Haar G. Therapeutic ultrasound. European Journal of Ultrasound, 1999, 9(1): 3-9.

[54] Umemura S, Kawabata K, Sasaki K, Yumita N, Umemura K and Nishigaki R. Recent advances in sonodynamic approach to cancer therapy. Ultrasonics Sonochemistry, 1996, 3: S187-S191.

[55] Ng KY and Liu Y. Therapeutic ultrasound: its application in drug delivery. Medicinal Research Reviews, 2002, 22(2): 204-223.

[56] Rychak JJ, Klibanov AL, Ley KF and Hossack JA. Enhanced targeting of ultrasound contrast agents using acoustic radiation force. Ultrasound in Medicine \& Biology, 2007, 33(7): 1132-1139.

[57] Frenkel V. Ultrasound mediated delivery of drugs and genes to solid tumors. Adv. Drug Deliv. Rev., 2008, 60: 1193-1208.

[58] Billard B, Hynynen K and Roemer R. Effects of physical parameters on high temperature ultrasound hyperthermia. Ultrasound in medicine \& biology, 1990, 16(4): 409-420.

[59] Partanen A, Yarmolenko PS, Viitala A, Appanaboyina S, Haemmerich D, Ranjan A, Jacobs G, Woods D, Enholm J and Wood BJ. Mild hyperthermia with magnetic resonance-guided high-intensity focused ultrasound for applications in drug delivery. International Journal of Hyperthermia, 2012, 28(4): 320-336.

[60] Chomas JE, Dayton P, Allen J, Morgan K and Ferrara KW. Optical observation of contrast agent destruction. Appl. Phys. Lett., 2000, 77: 1056-1058.

[61] Chomas JE, Dayton PA, May D, Allen J, Klibanov K and Ferrara KW. Optical observation of contrast agent destruction. Appl. Phys. Lett., 2000, 77: 1056-1058.

[62] Marmottant P, Meer Svd, Emmer M, Versluis M, Jong Nd, Hilgenfeldt S and Lohse D. A model for large amplitude oscillations of coated bubbles accounting for buckling and rupture. J. Acous. Soc. Am., 2005, 118(6): 3499-3505.

[63] Brennen CE. Cavitation and bubble dynamics, Oxford University Press, USA, 1995.

[64] Sboros V. Response of contrast agents to ultrasound. Advanced Drug Delivery Reviews, 2008, 60(10): $1117-1136$

[65] Ferrara KW. Driving delivery vehicles with ultrasound. Advanced Drug Delivery Reviews, 2008, 60: 1097-1102.

[66] Kimmel E. Cavitation bioeffects. Critical reviews in biomedical engineering, 2006, 34(2): 105.

[67] Miller MW, Miller DL and Brayman AA. A review of in vitro bioeffects of inertial ultrasonic cavitation from a mechanistic perspective. Ultrasound in Medicine \& Biology, 1996, 22(9): 1131-1154.

[68] Morgan KE, Allen JE, Dayton PA, Chomas JE, Klibaov AL and Ferrara KW. Experimental and theoretical evaluaiton of microbubble behavior: effect of transmitted phase and bubble size. IEEE transactions on Ultrasonics, Ferroelectrics and Frequency Control, 2000, 47(6): 1494-1509.

[69] Suslick KS. Sonochemistry. Science, 1990, 247(4949): 1439-1445.

[70] Larina I, Evers B, Bartels C, Ashitkov T, Larin K and Esenaliev R. Ultrasound-enhanced drug delivery for efficient cancer therapy. 2002, 1:492-493 vol. 1.

[71] Mehier-Humbert S, Bettinger T, Yan F and Guy RH. Plasma membrane poration induced by ultrasound exposure: implication for drug delivery. Journal of Controlled Release, 2005, 104: 213-222.

[72] Miller DL, Pislaru SV and Greenleaf JF. Sonoporation: mechanical DNA delivery by ultrasonic cavitation. Somatic cell and molecular genetics, 2002, 27(1): 115-134.

[73] Miller DL and Quddus J. Sonoporation of monolayer cells by diagnostic ultrasound activation of contrast-agent gas bodies. Ultrasound in medicine \& biology, 2000, 26(4): 661-667.

[74] Ohl CD, Arora M, Ikink R, De Jong N, Versluis M, Delius M and Lohse D. Sonoporation from jetting cavitation bubbles. Biophysical Journal, 2006, 91(11): 4285-4295.

[75] Qiu Y, Luo Y, Zhang Y, Cui W, Zhang D, Wu J, Zhang J and Tu J. The correlation between acoustic cavitation and sonoporation involved in ultrasound-mediated DNA transfection with polyethylenimine (PEI) in vitro. Journal of Controlled Release, 2010, 145: 40-48. 
[76] Ward M, Wu J and Chiu JF. Experimental study of the effects of optison ${ }^{\circledR}$ concentration on sonoporation in vitro. Ultrasound in Medicine \& Biology, 2000, 26(7): 1169-1175.

[77] Kuroki M, Hachimine K, Abe H, Shibaguchi H, Maekawa SI, Yanagisawa J, Kinugasa T, Tanaka T and Yamashita Y. Sonodynamic therapy of cancer using novel sonosensitizers. Anticancer Research, 2007, 27(6A): 3673-3677.

[78] Yumita N, Nishigaki R, Umemura K, Morse PD, Swartz HM, Cain CA and Umemura S. Sonochemical activation of Hematoporphyrin: an ESR study. Radiation Res., 1994, 138: 171-176.

[79] Larina IV, Evers BM, Ashitkov TV, Bartels C, VLarin KV and Esenaliev RO. Enhancement of drug delivery in tumors by using interaction of nanoparticles with ultrasound radiation. Technology in Cancer Research \& Treatment, 2005, 4(2): 217-226.

[80] Larina IV, Evers BM and Esenaliev RO. Optimal drug and gene delivery in cancer cells by ultrasoundinduced cavitation. Anticancer Research, 2005, 25: 149-156.

[81] Kopechek JA, Park E, Mei C-S, McDannold NJ and Porter TM. Accumulation of phase-shift nanoemulsions to enhance MR-guided ultrasound-mediated tumor ablation in vivo. Journal of Healthcare Engineering, 2013, 4(1): 109-126.

[82] Tartis MS, Kruse DE, Zheng HR, Zhang H, Kheirolomoom A, Marik J and Ferrara KW. Dynamic microPET imaging of ultrasound contrast agents and lipid delivery. J. Controlled Release, 2008, 131: $160-166$.

[83] Dromi S, Frenkel V, Luk A, Traughber B, Angstadt M, Bur M, Poff J, Xie JW, Libutti SK, Li KCP and Wood BJ. Pulsed-high intensity focused ultrasound and low temperature-sensitive liposomes for enhanced targeted drug delivery and antitumor effect. Clin. Cancer Res., 2007, 13(9): 2722-2727.

[84] Kataoka K, Matsumoto T, Yokoyama M, Okano T, Sakurai Y and Fukushima S. Doxorubicin-loaded poly(ethylene glycol)-poly(beta-benzyl-L-aspartate) copolymer micelles: their pharmaceutical characteristics and biological significance. J. Control Release, 2000, 64: 143-153.

[85] Husseini GA, Myrup G, Pitt WG, Christensen DA and Rapoport N. Factors affecting acoustically triggered release of drugs from polymeric micelles. J. Controlled Release, 2000, 69: 43-52.

[86] Rapoport N, Marin A, Luo Y, Prestwich G and Muniruzzaman M. Intracellular uptake and trafficking of pluronic micelles in drug-sensitive and MDR cells: effect on the intracellular drug localization. $J$. Pharm. Sci., 2002, 91: 157-170.

[87] Munshi N, Rapoport N and Pitt WG. Ultrasonic activated drug delivery from Pluronic P-105 micelles. Cancer Letters, 1997, 118: 13-19.

[88] Greenleaf WJ, Bolander ME, Sarkar G, Goldring MB and Greenleaf JF. Artificial cavitation nuclei significantly enhance acoustically induced cell transfection. Ultrasound in Medicine \& Biology, 1998, 24: 587-595.

[89] Uchida T, Tachibana K, Hisano S and Morioka E. Elimination of adult T cell leukemia cells by ultrasound in the presence of porfimer sodium. Anti-cancer Drugs, 1997, 8(4): 329-335.

[90] Xing Z, Wang J, Ke H, Zhao B, Yue X, Dai Z and Liu J. The fabricaiton of novel nanobubble ultrasound contrast agent for potential tumor imaging. Nanotechnology, 2010, 21: 145607.

[91] Cavalli R, Bisazza A, Trotta M, Argenziano M, Civra A, Donalisio M and Lembo D. New chitosan nanobubbles for ultrasound-mediated gene delivery: preparation and in vitro characterization. Int. J. Nanomedicine, 2012, 7: 3309-3318.

[92] Lapotko D. Plasmonic nanobubbles as tunable cellular probes for cancer theranostics. Cancers, 2011, 3(1): 802-840.

[93] Perez JLJ, Orea AC, Gallegos ER and Fuentes RG. Photoacoustic spectroscopy to determine in vitro the nonradiative relaxation time of porotoporphyrin IX solution containing gold metallic nanoparticles. Eur. Phys. J. Spec. Top, 2008, 152: 353-356.

[94] Yamaguchi S, Kobayashi H, Narita T, Kanehira K, Sonezaki S, Kudo N, Kubota Y, Terasaka S and Houkin K. Sonodynamic therapy using water-dispersed $\mathrm{TiO}_{2}$-polyethylene glycol compound on glioma cells: comparison of cytotoxic mechanism with photodynamic therapy. Ultrason. Sonochem., 2011, 18: 1197-1204. 
[95] Petty HR. Spatiotemporal chemical dynamics in living cells: From inforamtion trafficking to cell physiology. Biosystems, 2006, 83: 217-224.

[96] Park J, Fan Z, Kumon RE, El-Sayed MEH and Deng CX. Modulation of intracellular $\mathrm{CA}^{2+}$ concentration in brain microvascular endothelial cells in vitro by acoustic cavitation. Ultrasound in Medicine \& Biology, 2010, 36(7): 1176-1187.

[97] Zhong W, Sit WH, Wan JMF and Yu ACH. Sonoporation induces apoptosis and cell cycle arrest in human promyelocytic leukemia cells. Ultrasound in Medicine \& Biology, 2011, 37(12): 2149-2159.

[98] Guzman HR, Nguyen DX, McNamara AJ and Prausnitz MR. Equilibrium loading of cells with macromolecules by ultrasound: effects of molecular size and acoustic energy. J. Pharm. Sci., 2002, 91: 1693-1701.

[99] Alam MI, Beg S, Samad A, Baboota S, Kohli K, Ali J, Ahuja A and Akbar M. Strategy for effective brain drug delivery. European Journal of Pharmaceutical Sciences, 2010, 40(5): 385-403.

[100] Hynynen K, McDannold N, Vykhodtseva N and Jolesz FA. Noninvasive MR imaging-guided focal opening of the blood-brain barrier in rabbits. Radiology, 2001, 220: 640-646.

[101] McDannold N, Vykhodtseva N and Hynynen K. Effects of acoustic parameters and ultrasound contrast agent dose on focused-ultrasound induced blood-brain barrier disruption. Ultrasound in medicine \& biology, 2008, 34(6): 930-937.

[102] O'Reilly MA and Hynynen K. Ultrasound enhanced drug delivery to the brain and central nervous system. International Journal of Hyperthermia, 2012, 28(4): 386-396.

[103] Chen P-Y, Liu H-L, Hua M-Y, Yang H-W, Huang C-Y, Chu P-C, Lvu L-A, Tseng I-C, Feng L-Y, Tsai H-C, Chen S-M, Lu Y-J, Wang J-J, Yen T-C, Ma Y-H, Wu T, Chen J-P, Chuang J-I, Shin J-W, Hsueh $\mathrm{C}$ and Wei K-C. Novel magnetic/ultrasound focusing system enhances nanoparticle drug delivery for glioma treatment. Neuro. Oncol., 2010, 12(10): 1050-1060.

[104] Liu H-L, Hua M-Y, Chen P-Y, Chu P-C, Pan C-H, Yang H-W, Huang C-Y, Wang J-J, Yen T-C and Wei K-C. Blood-brain barrier disruption with focused ultrasound enhances delivery of chemotherapeutic drugs for glioblastoma treatment. Radiology, 2010, 255: 415-425.

[105] Langer R. Biomaterials in drug delivery and tissue engineering: one laboratory's experience. Acc. Chem. Res., 2000, 33: 94-101.

[106] Polat BE, Hart D, Langer R and Blankschtein D. Ultrasound-mediated transdermal drug delivery: Mechanism, scope, and emerging trends. Journal of Controlled Release, 2011, 152(3): 330-348. 


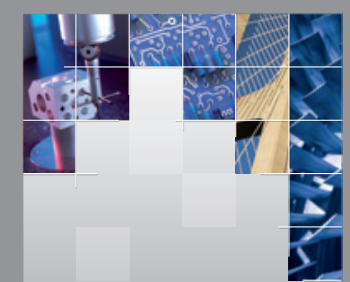

\section{Enfincering}
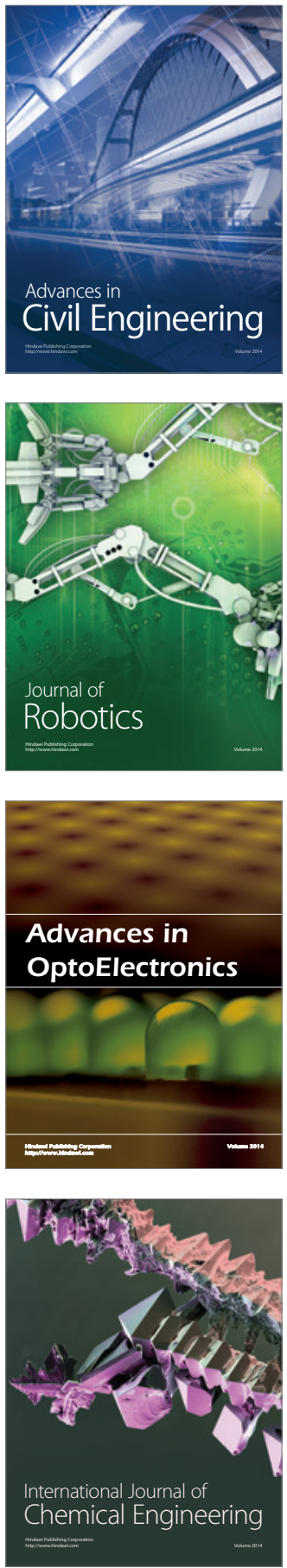

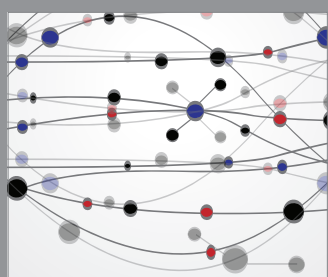

The Scientific World Journal

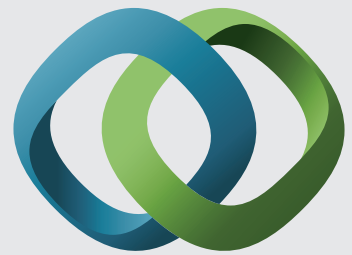

\section{Hindawi}

Submit your manuscripts at

http://www.hindawi.com
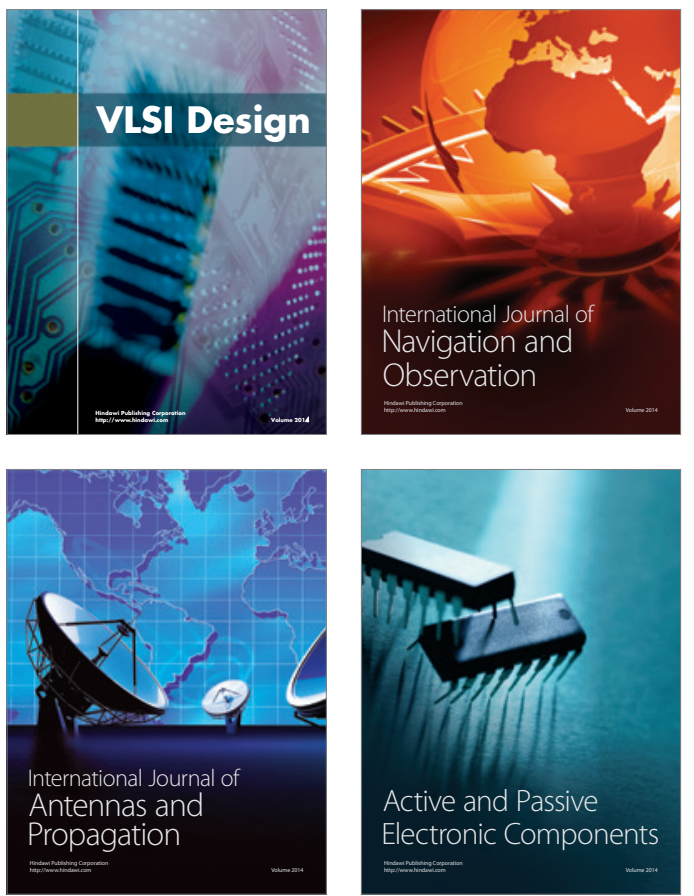
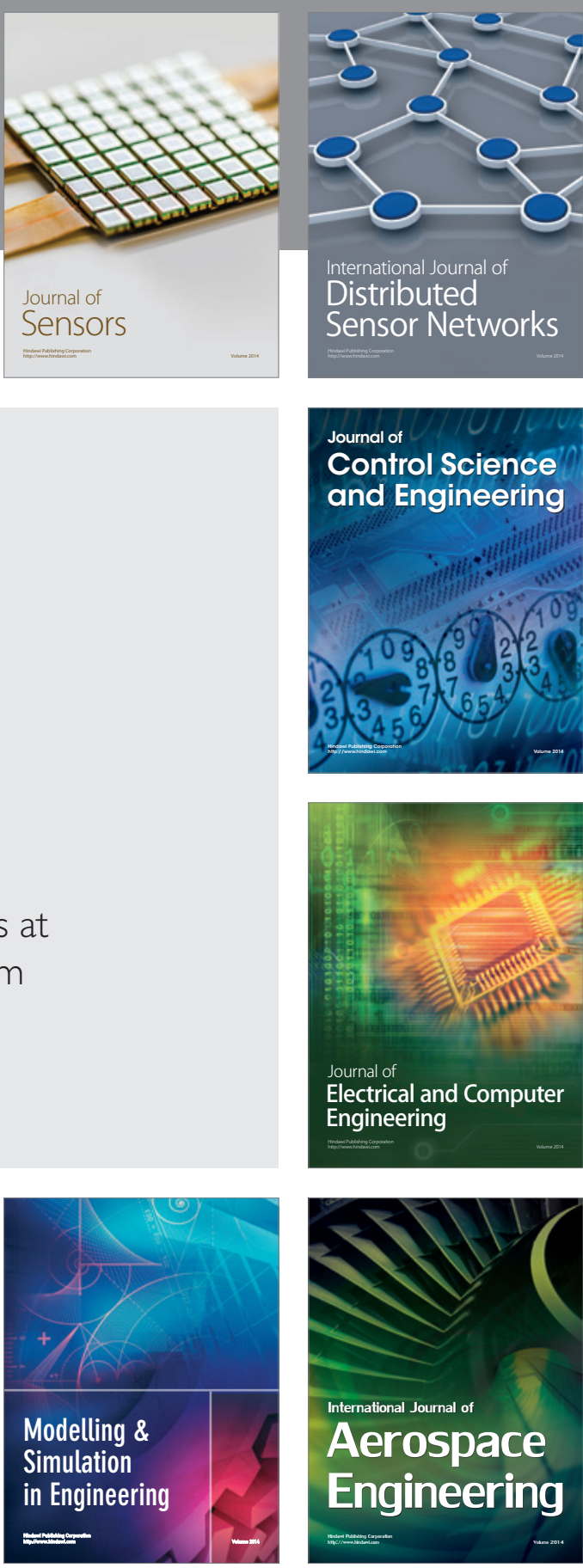

International Journal of

Distributed

Sensor Networks

Journal of

Control Science

and Engineering
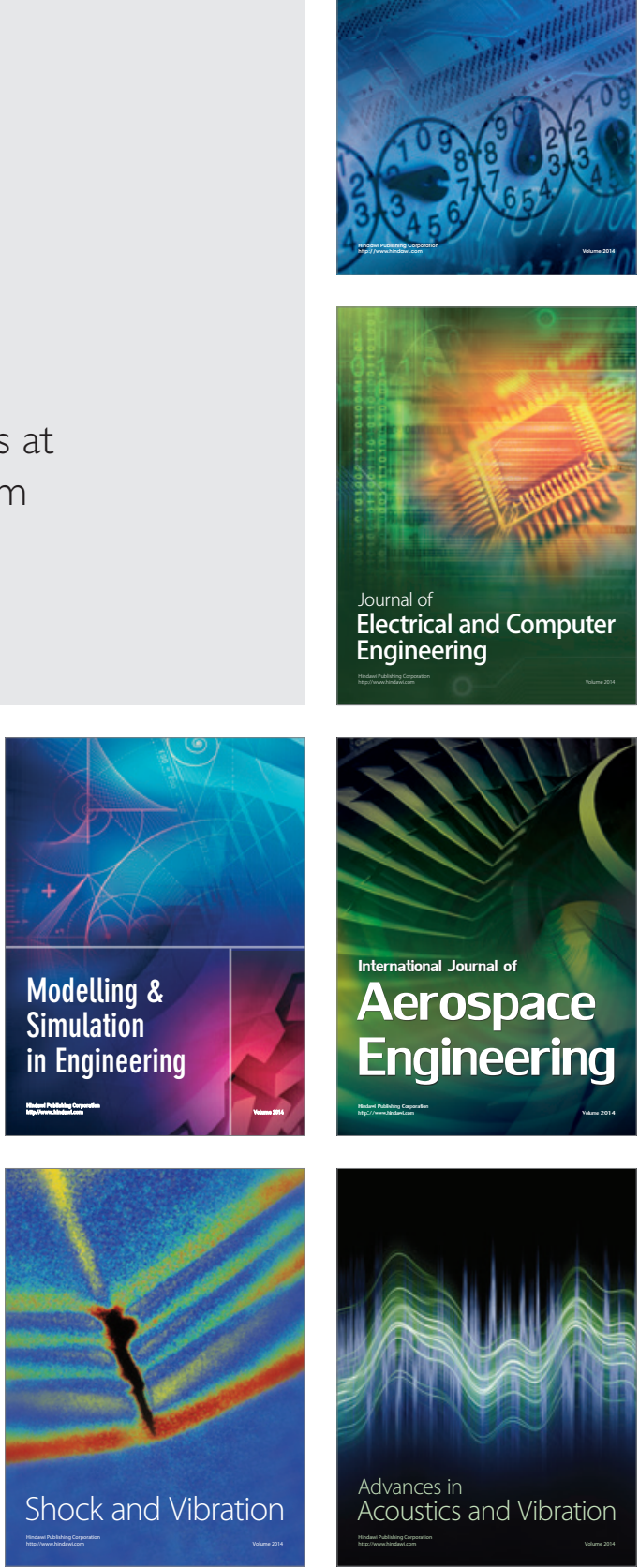